\title{
Feeding Polyunsaturated Supplements to Grazing Dairy Cows Improve the Healthy Value of Milk Fatty Acids
}

\author{
Gerardo Antonio Gagliostro ${ }^{*}$, Daniel Alberto Garciarena ${ }^{1}$, María Alejandra Rodriguez $^{2}$, \\ Liliana Elisabet Antonacci ${ }^{1}$ \\ ${ }^{1}$ Area de Producción Animal, Instituto Nacional de Tecnología Agropecuaria, Balcarce, Argentina \\ ${ }^{2}$ Centro de Investigaciones Tecnológicas de la Industria Láctea, Instituto Nacional de Tecnología Industrial, Buenos Aires, \\ Argentina \\ Email: ^gagliostro.gerardo@balcarce.inta.gob.ar
}

How to cite this paper: Gagliostro, G.A., Garciarena, D.A., Rodriguez, M.A. and Antonacci, L.E. (2017) Feeding Polyunsaturated Supplements to Grazing Dairy Cows Improve the Healthy Value of Milk Fatty Acids. Agricultural Sciences, 8, 759782.

https://doi.org/10.4236/as.2017.88057

Received: June 20, 2017

Accepted: August 15, 2017

Published: August 18, 2017

Copyright $\odot 2017$ by authors and Scientific Research Publishing Inc. This work is licensed under the Creative Commons Attribution International License (CC BY 4.0).

http://creativecommons.org/licenses/by/4.0/

(c) (i) Open Access

\begin{abstract}
The objective of the experiment was to improve the healthy value of milk fatty acids (FA) by feeding sunflower oil (SO) or sunflower seed (SS) combined or not with fish oil (FO) to grazing dairy cows. Four Holstein cows $(515 \pm 80 \mathrm{~kg}$ liveweight) in late lactation (230 days postpartum) fitted with ruminal cannulae were allotted to a $4 \times 4$ Latin square design with factorial arrangement of treatments: $\mathrm{SS}=1.9 \mathrm{~kg}$ dry matter $(\mathrm{DM}) / \mathrm{cow} / \mathrm{d}$ of SS; $\mathrm{SO}=0.8 \mathrm{~kg} / \mathrm{cow} / \mathrm{d}$ of $\mathrm{SO} ; \mathrm{SS}-\mathrm{FO}=\mathrm{SS}+0.24 \mathrm{~kg} / \mathrm{cow} / \mathrm{d} \mathrm{FO}$ and SO-FO $=\mathrm{SO}+0.24 \mathrm{~kg} / \mathrm{FO}$. Cows grazed a high quality pasture offered at $11 \mathrm{~kg} \mathrm{DM} /$ cow/day. After the afternoon milking cows also received $5.6 \mathrm{~kg} \mathrm{DM} /$ cow of corn silage and during each milking time cracked corn grain $(1.3 \mathrm{~kg} \mathrm{DM} / \mathrm{cow})$ mixed with a mineral-vitamin premix was fed. Oils and SS were introduced via ruminal cannulae and SS was fed roughly grounded. Milk yield tended $(p<0.07)$ to increase in SO treatments $(9.9 \mathrm{vs} 8.7 \mathrm{~kg} / \mathrm{d})$. Yields $(\mathrm{kg} / \mathrm{d})$ of fat corrected milk (FCM) (8.01 vs 6.37$)$ and milk fat $(0.27$ vs 0.191$)$ increased $(p<0.05)$ in SO diets and milk fat content was not affected. Milk protein concentration (40.5 vs 37.0 $\mathrm{g} / \mathrm{kg})$ and yield $(0.397 \mathrm{vs} 0.322 \mathrm{~kg} / \mathrm{d})$ were higher $(p<0.05)$ in SO without effects of FO or their interaction. Milk cholesterol content did not differ. The reduction in the atherogenic saturated $\mathrm{FA}$ of milk averaged $63 \%$ for $\mathrm{C}_{12: 0}, 51 \%$ for $\mathrm{C}_{14: 0}$ and $29 \%$ for $\mathrm{C}_{16: 0}$. Atherogenicity index (AI) of milk was reduced particularly in SS-FO. Basal concentration of cis-9, trans-11 $\mathrm{C}_{18: 2}$ (CLA) in milk was $1.39 \mathrm{~g} / 100 \mathrm{~g}$ FA and increased $(p<0.05)$ by $144 \%$ across treatments without differences between SS or SO. Feeding FO increased $(p<0.05)$ milk contents of CLA (2.86 to $3.92 \mathrm{~g} / 100 \mathrm{~g} \mathrm{FA}$ ) and linolenic acid. Comparing SO or SS with or without FO showed no changes for neutral detergent fiber (NDF) and crude protein (CP) degradation of pasture. Polyunsaturated fatty acid (PUFA) fed to grazing dairy cows had a marked effect on milk FA profile putatively
\end{abstract}


enhancing its healthy value.

\section{Keywords}

Grazing Dairy Cow, Milk Fatty Acids, Conjugated Linoleic Acid

\section{Introduction}

Milk fatty acid composition is a determinant factor of the healthy properties of milk owing to the potential effects of specific fatty acids on human health. Bovine milk fat represents up to $75 \%$ of the total consumption of fat from ruminal animals and dairy products provide about $25 \%-35 \%$ of the total saturated fat in the human diet [1]. When consumed in excess, some saturated fatty acids contained in milk fat (lauric $\left(\mathrm{C}_{12: 0}\right)$, myristic $\left(\mathrm{C}_{140}\right)$ and palmitic $\left(\mathrm{C}_{16: 0}\right)$ may be atherogenic [2] and are associated to increased risk of heart disease [1] [3]. Feeding PUFA rich supplements to dairy cows is an effective tool to inhibit de novo mammary synthesis of saturated fatty acids decreasing the atherogenic fraction of milk fat [1]. The inhibition tends to be higher when the number of carbon atoms and/or the degree of unsaturation of supplementary fatty acids increases [1]. A current special interest exists on conjugated linoleic acids (CLA) because they play an important role regulating levels of plasma lipids and cardiovascular functions, reducing cancer incidence, as well as blocking tumor growth and metastasis from breasts [3]. Vaccenic acid (trans-11C $\left.\mathrm{C}_{18: 1}, \mathrm{VA}\right)$, is the main natural trans fatty acid and precursor of the 9-cis, 11-trans $\mathrm{C}_{18: 2}$ CLA also called rumenic acid (RA) with anticarcinogenic properties itself and conversion to RA by human tissues [4]. Milk fat is considered the main natural source of RA and CLA and their concentration in milk is highly dependent on diet and lipid supplementation [1].

The range in CLA concentrations of milk from grazing cows is large (0.5 to $1.7 \%)$ due to seasonal changes in forage availability, botanical composition, pasture lipid and linolenic $\left(\mathrm{C}_{18: 3}\right)$ acid contents plus other unknown factors [1] [5]. Pasture-based diets results in milk with higher basal levels of CLA but further increases can be achieved by lipid supplementation [1] [5]. Dietary addition of PUFA rich-oils often results in substantial increases in milk CLA content with a linear effect of adding sunflower oil on milk CLA up to $4 \%$ of inclusion regarding total DM intake [1]. This was mainly explained by the increased availability of substrates for CLA or VA production in the rumen [1] [4]. Free oils are more effective to increase milk CLA and VA than oilseeds, due to the gradual release of PUFA from seeds compared to oils. This fact may allow a greater biohydrogenation of VA after seed feeding and hence a lower availability of the CLA precursor for absorption in the intestines [1]. Studies in goats revealed that $\mathrm{C}_{18: 2}$ contained in seed was more extensively hydrogenated to $\mathrm{C}_{18: 0}$ compared to $\mathrm{C}_{18: 2}$ contained in oil [1]. Direct comparisons between effects of oilseeds and free oils 
on milk fatty acid profile and CLA content in grazing dairy cows are lacking. The distinct and more effective effects of free oils should be weighed with the difficulty for the farmer to manipulate liquid (not-solid) supplements. In addition, it may also be a more expensive way to increase milk CLA considering the higher prices of oils than seeds and some detrimental effects of free oils on rumen function could also be expected.

Another strategy to enhance milk CLA content is feeding fish oil rich in longchain PUFA such as eicosapentaenoic acid $\left(\mathrm{C}_{20: 5 n 3}, \mathrm{EPA}\right)$ and docosahexaenoic acid $\left(\mathrm{C}_{22: 6 n 3}, \mathrm{DHA}\right)$ which may reduce microbial activity associated with the biohydrogenation pathway of CLA precursors [1]. One factor that may restrict the use of fish oil is its negative effect on the rumen environment and on the digestion of forage fiber (NDF) and protein [1] [4]. The objective of this study was to evaluate the effect of two sources of supplementary $\mathrm{C}_{18: 2}$ (sunflower seed vs sunflower oil) combined or not with fish oil on milk production and composition, milk fatty acid profile and ruminal degradation of forage fiber (NDF) and crude protein in grazing dairy cows.

\section{Materials and Methods}

\subsection{Cows and Treatments}

The experiment was conducted at the National Institute of Agricultural Technology (INTA) in Balcarce $\left(37^{\circ} 45^{\prime} \mathrm{S}, 58^{\circ} 18^{\prime} \mathrm{W}\right)$, Argentina, during 60 days at the end of the winter. Four multiparous Holstein cows (515 $\pm 80 \mathrm{~kg}$ liveweight) in late lactation (230 days postpartum) fitted with ruminal cannulae were used. Before the start of the experiment, animals received a pre-trial diet (Table 1) without supplemental lipids during two weeks to asses the basal milk fatty acid profile. After this phase, the cows were assigned to 4 dietary treatments (Table 1) in a $4 \times 4$ Latin square design with a factorial arrangement. Experimental periods lasted 15 days with the first 10 for adaptation and the last 5 for data collection. Cracked corn grain mixed with a mineral-vitamin premix (Table 1) were

Table 1. Feed ingredients for the pre-trial and the experimental treatments diets.

\begin{tabular}{cccccc}
\hline Ingredients, kg DM/cow/d & Pre-trial & & SO & SS-FO & SO-FO \\
\hline Ground corn & 2.6 & 2.6 & 2.6 & 2.6 & 2.6 \\
Sunflower seed (SS) & - & 1.9 & - & 1.9 & - \\
Sunflower meal & 0.9 & - & 0.9 & - & 0.9 \\
Sunflower oil (SO) & - & - & 0.8 & - & 0.88 \\
Fish oil (FO) & - & - & - & 0.24 & 0.24 \\
Corn silage & 5.6 & 5.6 & 5.6 & 5.6 & 5.6 \\
Mineral-vitamin premix ${ }^{(1)}$ & 0.25 & 0.25 & 0.25 & 0.25 & 0.25 \\
Pasture offered & 11.0 & 11.0 & 11.0 & 11.0 & 11.0 \\
\hline
\end{tabular}

${ }^{(1)}$ Contained (wt/wt) 21\% $\mathrm{Ca}_{3}\left(\mathrm{PO}_{4}\right)_{2}, 4.6 \% \mathrm{P}_{2} \mathrm{O}_{5}, 6 \% \mathrm{MgO}, 3 \%$ molasses, $0.15 \% \mathrm{Fe} \mathrm{SO}_{4}, 0.4 \% \mathrm{CuSO}, 0.3 \%$ $\mathrm{Zn} \mathrm{SO}, 0.04 \% \mathrm{MnSO}_{4}, 0.02 \% \mathrm{CoSO}_{4}, 0.02 \% \mathrm{Na}_{2} \mathrm{SeO}_{3}, 0.01 \% \mathrm{I}_{2}$, vitamin A (6,500,000 IU/kg), vitamin $\mathrm{D}_{3}$ $(1,600,000 \mathrm{IU} / \mathrm{kg})$, vitamin E (12,500 IU/kg), 64.46\% excipient. 
fed during each milking time. After milkings, the half daily dose of sunflower seed, sunflower oil and fish oil was introduced via ruminal cannulae in a single dose-pulse to simulate a rapid ingestion of lipids and to avoid refusals. Sunflower seed was previously grounded to allow a rapid release of fatty acids into the rumen. The sunflower oil dose was calculated to approximately provide $4.7 \%$ of the total DM intake registered in a previous experiment [6] whereas fish oil dose was fixed to represent about $1.4 \%$ of DM intake.

After the morning milking (08.00) and until the afternoon one (16.00), the cows were included into one of 4 grazing groups of cows from a whole experiment including 64 cows according to treatments. Pasture was strip-grazed managed. The area of each strip was adjusted in order to achieve an herbage allowance of $11 \mathrm{~kg} \mathrm{DM} /$ cow per d. After the afternoon milking the cows were offered corn silage at $5.6 \mathrm{~kg} \mathrm{DM} /$ cow. Treatments that included sunflower oil were balanced for protein by adding sunflower meal $(0.9 \mathrm{~kg} \mathrm{DM} / \mathrm{cow}$ per day) to the corn silage (Table 1).

\subsection{Samples Collection and Analyses}

Milk production was individually recorded over the last $5 \mathrm{~d}$ of each experimental period. Milk samples were collected at $\mathrm{d} 12^{\text {th }}$ and $15^{\text {th }}$ of each period, composited according to the corresponding volume measured at each milking time and analyzed for fat, protein and lactose by infrared spectrophotometry (Foss 300 Milko-Scan, Foss Electric, Hillerфd, Denmark). Milk urea nitrogen (MUN) and cholesterol were determined using commercial enzymatic kits (Wiener Laboratory, Rosario, Argentina). Milk fatty acid composition was measured on each cow before the start of lipid supplementation to obtain the basal profile in a sample collected at the end of the pre-trial period and also in pooled samples at d $12^{\text {th }}$ and $15^{\text {th }}$ of each experimental period. Fat was extracted from raw milk with a warm detergent Triton solution that was a mixture of $12 \mathrm{ml}$ of Triton $\mathrm{X}-100,50 \mathrm{ml}$ of isopropylic alcohol, $2.5 \mathrm{~g}$ of urea, $25 \mathrm{~g}$ of sodium hexametaphosphate and distilled water. The extraction was done in an oven at $90^{\circ} \mathrm{C}$. The hydrophobic upper layer was separated from the aqueous layer and transferred into a vial. Total lipids from the diet ingredients (pasture, sunflower oil, sunflower seed, corn silage and corn grain) were extracted in a cartridge over a Twisselman extractor by adding $50 \mathrm{ml}$ of hexane to each $5 \mathrm{~g}$ of sample. Extraction was fulfilled by heating during 2 hours. Solvent was recovered by distillation. For the esterification and methylation of fatty acids, methyl esters of fatty acids were obtained either from feedstuffs or from milk fat by adding $5 \mathrm{ml}$ of hexane, mixing in vortex. It was added $0.2 \mathrm{ml}$ of esterificant reactives, mixed in vortex for 1 minute, leaving in the vial for 5 minutes. Latter on added $0.5 \mathrm{~g}$ of sodium sulfate acid monohydrate and mixed using a vortex and centrifuged ( 3 min at $2500 \mathrm{rpm}$ ). The supernatant was transferred to a glass vial and injected in a gas-liquid chromatograph (GLC). Chromatography was performed using a ca- 
pillary column (Varian WCOT-100M, $100 \mathrm{~m} \times 0.25 \mathrm{~mm} \times 0.25 \mu \mathrm{m}$ ) in an Agilent 6890 series plus. One $\mu \mathrm{l}$ of each sample was injected. The initial oven temperature was held at $70^{\circ} \mathrm{C}$ for 1 minute, increased $5^{\circ} \mathrm{C} / \mathrm{min}$ to $100^{\circ} \mathrm{C}$, then, increased $10^{\circ} \mathrm{C} / \mathrm{min}$ to $160^{\circ} \mathrm{C}$, increased $5^{\circ} \mathrm{C} / \mathrm{min}$ to $175^{\circ} \mathrm{C}$, and increased $5^{\circ} \mathrm{C} /$ min to final temperature of $225^{\circ} \mathrm{C}$. Hidrogen was the gas carrier, the flow was held at $1.4 \mathrm{~mL} / \mathrm{min}$. The injector temperature was $250^{\circ} \mathrm{C}$ and the FID detector temperature was maintained at $355^{\circ} \mathrm{C}$. Individual CLA isomers (cis-9, trans$11 \mathrm{C}_{18: 2}$; trans-10, cis-12 $\mathrm{C}_{18: 2}$, trans-9, trans-11C $\mathrm{C}_{18: 2} ;$ cis-9, cis-11 $\mathrm{C}_{18: 2}$ ) were identified based on individual standards (Matreya, Inc. Cat\# 1255; 1254; 1257; 1256), whereas the individual standards of trans- $\mathrm{C}_{18: 1}, \mathrm{C}_{20: 4}, \mathrm{C}_{20: 5}$ and $\mathrm{C}_{22: 6}$ were purchased at Sigma (cat\# V1381; A9298; E2012; D2659).

Pasture intake was estimated by the difference between the herbage offered minus the herbage refused [7]. Pasture samples were obtained by hand-plucking at the grazing height during intake measurements. Samples of concentrates and corn silage were collected every $7 \mathrm{~d}$. Forage and concentrate samples were dried in a forced-air oven $\left(60^{\circ} \mathrm{C}\right)$ during $36 \mathrm{~h}$, ground through a $1 \mathrm{~mm}$ screen (Wiley mill, Philadelphia, PA), and analyzed for DM, organic matter (OM), NDF and acid detergent fiber (ADF) [8], CP (Leco FP-528 autoanalizer), in vitro OM digestibility (IVDMO), water-soluble carbohydrate (WSC) [9] and ether extract (EE) [10]. Corn grain and maize silage were also assayed for starch content [11] and all feed samples were analyzed for fatty acid composition.

\subsection{Rumen Environment and In Situ Pasture Fiber and Protein Degradability}

During the $d 15^{\text {th }}$ of each experimental period samples of ruminal content were taken from the dorsal, ventral and caudal areas in the morning and in the afternoon at 3 and $6 \mathrm{~h}$ after the daily dose of sunflower seed, sunflower oil and fish oil. Ruminal fluid was obtained by straining through four layers of cheesecloth and $\mathrm{pH}$ was immediately measured (Orion portable $\mathrm{pH}$ meter $250 \mathrm{~A}$, Orion $\mathrm{Re}$ search Inc., Boston, USA). On day $13^{\text {th }}$ of each experimental period, samples of fresh pasture were obtained by hand-plucking and cut to a final length of $1 \mathrm{~cm}$. The wet material was immediately placed (approximately $4 \mathrm{~g}$ of DM/bag) in dacron bags $(15.5 \times 7.5 \mathrm{~cm}, 52 \mu \mathrm{m}$ pore size, Ankom Corp., Fairport, NY) and suspended in the ventral sac of the rumen. Triplicate bags were removed at 0,3 , $6,12,24,48$ and 72 hours. After incubation, the bags were rinsed in a pipette washer for 1 hour and then hand-washed with cold tap water. Bags were squeezed until the water was clear and then oven dried at $60^{\circ} \mathrm{C}$ until constant weight. The residues from each bag were weighed, ground through a $1 \mathrm{~mm}$ screen, pooled within cow for each time of incubation and analyzed for NDF and CP content. Rumen disappearances of CP and NDF were estimated from the initial and final quantities of each component. Kinetic parameters of ruminal NDF and CP degradation were estimated using the solver routine of Excel with the equation proposed by [12]: $\mathrm{D}=\mathrm{A}+\mathrm{B}(1-\mathrm{e}-\mathrm{kdt})$, where $\mathrm{D}=$ disappearance at time $(\mathrm{t}), \mathrm{A}=$ 
soluble fraction $(\%$, wash value at $0 \mathrm{~h}), \mathrm{B}=$ insoluble potentially digestible fraction $(\%), \mathrm{kd}=$ fractional rate of degradation $(\% / \mathrm{h})$, and $\mathrm{t}=$ time of incubation (h). Total potentially degradable fraction of NDF was estimated as $\mathrm{A}+\mathrm{B}$. In the estimation of NDF degradation the model was forced to obtain a soluble (A) fraction equal or higher than zero.

\subsection{Statistical Analyses}

Data of milk production and composition and kinetic parameters of pasture NDF and CP degradation were analyzed as a $4 \times 4$ Latin square with factorial arrangements of treatments $(2 \times 2)$ using the GLM procedure of SAS (1995). The model used was: $Y_{i j k l}=\mu+P_{i}+C_{j}+S_{k}+F_{1}+S^{\star} F_{k l}+e_{i j k l}$, where $Y_{i j k l}$ is the dependent variable, $\mu$ is the overall mean, $P_{i}$ is the period effect, $C_{j}$ is the cow effect, $S_{k}$ is the source of linoleic acid effect (sunflower seed vs sunflower oil), $F_{1}$ is the fish oil effect, $\mathrm{S}^{\star} \mathrm{F}_{\mathrm{kl}}$ is the interaction effect and $\mathrm{e}_{\mathrm{ijk}}$ is the residual error. When the $\mathrm{S}^{\star} \mathrm{F}_{\mathrm{kl}}$ interaction resulted significant, means were compared using the Duncan's Multiple Range Test. Differences were considered significant with $p<0.05$ unless otherwise stated. Values are presented as least squares means (LSmeans) followed by standard error of the mean (SEM).

Rumen $\mathrm{pH}$ was analyzed using the model: $\mathrm{Y}_{\mathrm{ijkl}}=\mu+\mathrm{T}_{\mathrm{i}}+\mathrm{C}_{(\mathrm{i}) \mathrm{j}}+\mathrm{P}_{\mathrm{k}}+\mathrm{H}_{1}+\mathrm{T} \times$ $\mathrm{H}_{\mathrm{il}}+\mathrm{e}_{\mathrm{ijk} \mathrm{k}}$, where $\mathrm{Y}_{\mathrm{ijk}}=$ the dependent variable, $\mu=$ the population mean, $\mathrm{T}_{\mathrm{i}}=$ the effect of the ith treatment, $C_{(i) j}=$ the random effect of the $j_{\text {th }}$ cow within the $i_{\text {th }}$ treatment, $\mathrm{P}_{\mathrm{k}}=$ the effect of the $\mathrm{k}_{\mathrm{th}}$ period, $\mathrm{H}_{\mathrm{l}}=$ the effect of the $\mathrm{l}_{\mathrm{th}}$ hour, $\mathrm{T} \times \mathrm{H}_{\mathrm{il}}$ the effect of treatment $x$ hour interaction and $e_{i j k l}$ is the residual error. The $\mathrm{C}_{(\mathrm{i}) \mathrm{j}}$ term was used as an error term to test the $\mathrm{T}_{\mathrm{i}}$ effect.

\section{Results}

Dry matter content of pastures ranged from $18.9 \%$ to $24.4 \%$ throughout the experiment with moderate levels of crude protein $(12 \%-21.8 \%)$ and fiber $(35.4 \%$ $42.7 \%$ ). Pasture organic matter digestibility ranged from $72.5 \%$ to $84.9 \%$ and concentration of water soluble carbohidrates from 12.1 to 22.9. Levels of ether extract resulted high and were comprised between 6\% - 10\% (Table 2).

Corn silage was characterized by a high dry matter content with moderate fiber and starch and a low energy density compared to concentrates (Table 3).

Table 2. Chemical composition and in vitro OM digestibility (IVOMD) of the pasture during the experiment ${ }^{(1)}$.

\begin{tabular}{ccccccccc}
\hline & \multicolumn{7}{c}{ \%, DM basis } \\
\hline & \% DM & IVOMD $^{(2)}$ & OM & CP & NDF & ADF & WSC $^{(3)}$ & EE \\
\hline Average & 21.2 & 76.7 & 91.5 & 16.1 & 38.3 & 23.7 & 17.5 & 7.6 \\
SD & 2.2 & 5.6 & 1.8 & 4.1 & 3.5 & 2.3 & 8.2 & 2.1 \\
Minimum & 18.9 & 72.5 & 89.9 & 12 & 35.4 & 20.4 & 12.1 & 6.0 \\
Maximum & 24.4 & 84.9 & 93.7 & 21.8 & 42.7 & 25.8 & 22.9 & 10.0 \\
\hline
\end{tabular}

${ }^{(1)}$ Samples were obtained by hand plucking. ${ }^{(2)}$ as $\%$ of OM. ${ }^{(3)} \mathrm{WSC}=$ water-soluble carbohydrates and EE = ether extract. 
Table 3. Chemical composition and in vitro OM digestibility (IVOMD) of corn silage and concentrates.

\begin{tabular}{ccccc}
\hline & \multicolumn{3}{c}{ Feeds } \\
\cline { 2 - 5 } & Corn silage & Corn grain & Sunflower meal & Sunflower seed \\
\hline DM, \% & 37.6 & 86.9 & 89.8 & 94.4 \\
OM, \% DM & 93.5 & 98.1 & 91.9 & 96.5 \\
IVOMD, \% & 65.2 & 90.1 & 81.1 & \\
CP, \% DM & 6.8 & 5.9 & 38.4 & 11.1 \\
NDF, \% DM & 40 & 11.3 & 27.1 & 38.2 \\
ADF, \% DM & 25.6 & 4.7 & 20.3 & 30.9 \\
WSC (1), \% DM & 18.2 & 18.3 & 9.5 & 3.9 \\
Starch, \% DM & 26.4 & 71.9 & 1.2 & 0.7 \\
Ether extract, \% DM & 6.4 & 5.1 & 2.5 & 42.6 \\
Metabolizable energy, & 2.3 & 3.2 & 3.6 & 4.7 \\
Mcal/kg DM & & & &
\end{tabular}

${ }^{(1)}$ Water-soluble carbohydrates.

Sunflower meal was rich in protein (38.4\%) and contributed to keep diets isonitrogenous (Table 6).

Fatty acid profile of feeds and oils is given in Table 4. Pasture represented a good source of $\mathrm{C}_{18: 3 n-3}$ whereas corn silage and grain and sunflower meal, seed and oil were sources $\mathrm{C}_{18: 2 \mathrm{n}-6}$ for milk CLA precursors and synthesis. As expected, fish oil furnished the long chain supplementary $\mathrm{n}-3$ acids (EPA and DHA, Table 4).

Total DM intake of cows averaged $14.89 \mathrm{~kg} / \mathrm{d}$ and pasture intake ranged from 5.15 to $6.26 \mathrm{~kg}$ DM. Intake of $\mathrm{C}_{18: 2}$ and $\mathrm{C}_{18: 3}$ was calculated to be 0.708 and 0.128 $\mathrm{kg} /$ cow respectively (Table 5 ).

Pasture intake represented from $37.3 \%$ (sunflower oil treatment) to $41.4 \%$ (sunflower seed) of total dry matter intake of cows while sunflower oil and fish oil represented about $5.6 \%$ and $1.6 \%$ respectively (Table 6 ). Similar values for metabolic energy (2.98 Mcal/kg MS), EE (12.2\%), crude protein (11.3\%) and fiber (34.5\%) contents of rations were observed (Table 6).

\section{Milk Production and Composition}

Effects of fish oil supply or interaction were not detected (Table 7). Milk yield tended $(p<0.07)$ to increase in sunflower oil treatments $(9.9 \mathrm{vs} 8.7 \mathrm{~kg} / \mathrm{d}$ ) while fat corrected milk ( 8.01 vs $6.37 \mathrm{~kg} / \mathrm{d}$ ) and milk fat yield (270 vs $191 \mathrm{~g} / \mathrm{d}$ ) increased in sunflower oil diets. Milk fat content resulted low in all groups and not affected by treatments. Milk protein concentration ( $40.5 \mathrm{vs} 37.0 \mathrm{~g} / \mathrm{kg}$ ) and yield ( 397 vs $322 \mathrm{~g} / \mathrm{d}$ ) were higher in sunflower oil diets. Lactose content was higher in diets including sunflower oil ( $48.5 \mathrm{vs} 45.7 \mathrm{~g} / \mathrm{kg}$ ) as the consequence of both, the highest concentration observed in sunflower oil-fish oil and the lower 
Table 4. Fatty acid (FA) content of feeds and oils used in the experiment.

\begin{tabular}{|c|c|c|c|c|c|c|c|}
\hline $\begin{array}{l}\text { Fatty acids, } \\
\text { g/100g total FA }\end{array}$ & Pasture & $\begin{array}{l}\text { Corn } \\
\text { grain }\end{array}$ & $\begin{array}{l}\text { Corn } \\
\text { silage }\end{array}$ & $\begin{array}{c}\text { Sunflower } \\
\text { meal }\end{array}$ & $\mathrm{SS}^{(1)}$ & $\mathrm{FO}^{(2)}$ & $\mathrm{SO}^{(3)}$ \\
\hline $\mathrm{C}_{12: 0}$ & & & 3.29 & & & & \\
\hline $\mathrm{C}_{14: 0}$ & 0.55 & & 6.24 & & & 3.77 & \\
\hline \multicolumn{8}{|l|}{$\mathrm{C}_{14: 1}$} \\
\hline $\mathrm{C}_{15: 0}$ & 0.06 & & & & & 0.62 & \\
\hline $\mathrm{C}_{15: 1}$ & & & & & & 0.40 & \\
\hline $\mathrm{C}_{16: 0}$ & 12.94 & 13.27 & 23.76 & 8.00 & 6.6 & 19.02 & 5.8 \\
\hline $\mathrm{C}_{16: 1}$ & 2.58 & 0.38 & & & & 5.78 & \\
\hline \multicolumn{8}{|l|}{$\mathrm{C}_{16: 2}$} \\
\hline \multicolumn{8}{|l|}{$\mathrm{C}_{16: 3}$} \\
\hline $\mathrm{C}_{17: 0}$ & & & & & & 0.45 & \\
\hline $\mathrm{C}_{17: 1}$ & & & & & & 0.45 & \\
\hline $\mathrm{C}_{18: 0}$ & 1.50 & 3.24 & 7.78 & 4.08 & 3.34 & 3.06 & 3.2 \\
\hline $\mathrm{C}_{18: 1}$ & 4.12 & 32.1 & 27.91 & 20.44 & 18.5 & 24.57 & 32.6 \\
\hline $\mathrm{C}_{18: 2 \mathrm{c} 9, \mathrm{c} 12}$ & 9.24 & 49.27 & 34.80 & 64.17 & 70.3 & 17.04 & 55.2 \\
\hline $\mathrm{C}_{18: 3 \mathrm{c} 9, \mathrm{c} 12, \mathrm{c} 15}$ & 56.54 & 1.76 & & 0.50 & 0.16 & 1.49 & 0.3 \\
\hline $\mathrm{C}_{18: 4 \mathrm{n} 3}$ & 0.48 & & & & & 2.58 & \\
\hline $\mathrm{C}_{20: 4} \Delta 5$ & & & & & & 1.12 & \\
\hline $\mathrm{C}_{20: 5} \Delta 5(\mathrm{EPA})^{(4)}$ & & & & & & 6.71 & \\
\hline $\mathrm{C}_{22: 6} \Delta 4(\mathrm{DHA})^{(5)}$ & & & & & & 11.02 & \\
\hline Total SFA ${ }^{(6)}$ & & & & & & 26.92 & 9.00 \\
\hline Total UFA ${ }^{(7)}$ & & & & & & 71.16 & 88.1 \\
\hline$n-6 / n-3$ & 0.16 & 27.9 & 34.8 & 128.3 & 439 & 0.74 & 184 \\
\hline
\end{tabular}

${ }^{(1)} \mathrm{SS}=$ sunflower seed, ${ }^{(2)} \mathrm{FO}=$ fish oil, ${ }^{(3)} \mathrm{SO}=$ sunflower oil. ${ }^{(4)}$ Ecosapentaenoic acid $\left(\mathrm{C}_{20,5}\right.$ c5,c8,c11,c14,c17$)$, ${ }^{(5)}$ Docosahexaenoic acid $\left(\mathrm{C} 22: 6_{\mathrm{c} 4, \mathrm{c}, \mathrm{c}, \mathrm{l} 1, \mathrm{c} 13, \mathrm{c}, \mathrm{l} 1, \mathrm{c} 19),}{ }^{(6)} \mathrm{SFA}=\right.$ saturated fatty acids, ${ }^{(7)} \mathrm{UFA}=$ unsaturated fatty acids.

Table 5. Dry matter (DM) and estimated fatty acid (FA) intakes in grazing dairy cows supplemented with sunflower seed (SS), sunflower oil (SO), sunflower seed plus fish oil (SS-FO) and sunflower oil plus fish oil (SO-FO).

\begin{tabular}{|c|c|c|c|c|}
\hline \multirow[b]{2}{*}{ Item. } & \multicolumn{4}{|c|}{ Treatment } \\
\hline & SS & SO & SS-FO & $\mathrm{SO}-\mathrm{FO}$ \\
\hline \multicolumn{5}{|l|}{ Intake, $\mathrm{kg} \mathrm{DM} / \mathrm{d}$} \\
\hline Pasture $^{(1)}$ & 6.26 & 5.15 & 5.66 & 5.64 \\
\hline Corn silage & 5.60 & 5.60 & 5.60 & 5.60 \\
\hline Corn grain & 1.35 & 1.36 & 1.74 & 1.37 \\
\hline Sunflower meal & - & 0.89 & - & 0.89 \\
\hline Sunflower seed & 1.89 & & 1.89 & \\
\hline Sunflower oil & & 0.80 & & 0.80 \\
\hline Fish oil & & & 0.24 & 0.24 \\
\hline Total & 15.1 & 13.8 & 15.13 & 15.54 \\
\hline \multicolumn{5}{|l|}{ Estimated fatty acid intake } \\
\hline $\mathrm{C}_{18: 2 \mathrm{c} 9, \mathrm{c} 12}, \mathrm{~g} / \mathrm{d}$ & 746 & 621 & 795 & 671 \\
\hline $\mathrm{C}_{18: 3 \mathrm{c} 9, \mathrm{c} 12, \mathrm{c} 15}, \mathrm{~g} / \mathrm{d}$ & 138 & 115 & 129 & 129 \\
\hline $\mathrm{C}_{18: 2 c 9, \mathrm{cl} 2}, \%$ total DM intake & 4.93 & 4.48 & 5.24 & 4.60 \\
\hline $\mathrm{C}_{18: 3 \mathrm{c} 9, \mathrm{c} 12, \mathrm{c} 15}, \% \mathrm{DM}$ & 0.91 & 0.83 & 0.85 & 0.89 \\
\hline $\mathrm{C} 20: 5 \Delta 5(\mathrm{EPA})^{(2)}, \mathrm{g} / \mathrm{d}$ & & & \multicolumn{2}{|c|}{16} \\
\hline $\mathrm{C} 22: 6 \Delta 4(\mathrm{DHA})^{(3)}, \mathrm{g} / \mathrm{d}$ & & & \multicolumn{2}{|c|}{26} \\
\hline
\end{tabular}

${ }^{(1)}$ Pasture fatty acid content averaged $50 \%$ of ether extract. ${ }^{(2)}$ Ecosapentaenoic acid $\left(\mathrm{C} 20: 5_{\mathrm{c} 5, \mathrm{c} 8, \mathrm{ccl} 1 \mathrm{c}, \mathrm{c} 4, \mathrm{cl} 7}\right)$,

${ }^{(3)}$ Docosahexaenoic acid $\left(\mathrm{C}_{22: 6 \mathrm{c} 4, \mathrm{c} 7, \mathrm{c} 10, \mathrm{c} 13, \mathrm{c} 19, \mathrm{c} 19}\right)$. 
Table 6. Total diet composition in grazing dairy cows supplemented with sunflower seed (SS), sunflower oil (SO), sunflower seed plus fish oil (SS-FO) and sunflower oil plus fish oil (SO-FO).

\begin{tabular}{ccccc}
\hline & \multicolumn{5}{c}{ Treatment } \\
\hline Item. & SS & SO & SS-FO & SO-FO \\
\hline Feeds, \% of total DM intake & & & & \\
Pasture & 41.4 & 37.3 & 37.4 & 38.7 \\
Corn silage & 37.2 & 40.7 & 37.1 & 38.6 \\
Corn grain & 8.9 & 9.8 & 11.5 & 9.4 \\
Sunflower meal & & 6.5 & & 6.2 \\
Sunflower seed & 12.5 & & 12.4 & \\
Sunflower oil & - & 5.8 & - & 5.5 \\
Fish oil & - & - & 1.6 & 1.6 \\
Nutrient composition ${ }^{(1)}$ & & & & 11.8 \\
CP, \% DM & 11.1 & 11.8 & 10.6 & 32.9 \\
NDF, \% DM & 36.5 & 33.4 & 35.2 & 13.1 \\
EE ${ }^{(2)}$ \% DM & 11.3 & 11.8 & 12.7 & 3.05 \\
\hline
\end{tabular}

${ }^{(1)}$ Estimated from the chemical composition of feeds (Table 2 and Table 3) and DM intake (Table 5). ${ }^{(2)} \mathrm{EE}=$ ether extract.

Table 7. Milk yield and composition in grazing dairy cows supplemented with sunflower seed (SS), sunflower oil (SO), sunflower seed plus fish oil (SS-FO) and sunflower oil plus fish oil (SO-FO).

\begin{tabular}{ccccccccc}
\hline & \multicolumn{3}{c}{ Treatment $^{(1)}$} & & \multicolumn{3}{c}{$\mathrm{P}<{ }^{(2)}$} \\
\hline Item. & SS & SO & SS-FO & SO-FO & SEM & A & B & $\mathrm{A} \times \mathrm{B}$ \\
\hline Milk, kg/d & 8.90 & 10.10 & 8.60 & 9.70 & 0.53 & 0.07 & 0.47 & 0.97 \\
$4 \% \mathrm{FCM}^{(3)}, \mathrm{kg} / \mathrm{d}$ & 6.90 & 8.10 & 5.80 & 7.90 & 0.63 & 0.04 & 0.39 & 0.47 \\
Fat, g/kg & 25.00 & 24.80 & 19.10 & 27.80 & 2.70 & 0.17 & 0.63 & 0.16 \\
Protein, g/kg & 38.70 & 40.70 & 35.40 & 40.4 & 0.90 & 0.01 & 0.11 & 0.15 \\
Lactose, g/kg & $48.20 \mathrm{~b}$ & $47.80 \mathrm{bc}$ & $43.20 \mathrm{c}$ & $49.20 \mathrm{a}$ & 0.80 & 0.02 & 0.07 & 0.01 \\
Protein/Fat & 1.58 & 1.70 & 1.94 & 1.57 & 0.22 & 0.59 & 0.64 & 0.31 \\
Fat, g/day & 221 & 267 & 161 & 273 & 30.70 & 0.04 & 0.40 & 0.32 \\
Protein, g/day & 341 & 406 & 303 & 388 & 21.8 & 0.01 & 0.24 & 0.65 \\
Cholesterol, mg/dl & 2.88 & 2.44 & 2.46 & 2.41 & 0.16 & 0.18 & 0.22 & 0.27 \\
Urea, mg/dl & 2.01 & 1.95 & 2.18 & 1.93 & 0.04 & 0.02 & 0.14 & 0.09 \\
\hline
\end{tabular}

${ }^{(1)}$ Least squares means \pm standard error of the mean (SEM). ${ }^{(2)}$ Duncan's Multiple Range Test $\mathrm{A}=(\mathrm{SS}+$ $\mathrm{SS}-\mathrm{FO})$ vs $(\mathrm{SO}+\mathrm{SO}-\mathrm{FO}) ; \mathrm{B}=(\mathrm{SS}+\mathrm{SO})$ vs $(\mathrm{SS}-\mathrm{FO}+\mathrm{SO}-\mathrm{FO}) \cdot{ }^{(3)} \mathrm{FCM}=4 \%$ fat corrected milk.

value registered in sunflower seed-fish oil. Milk urea nitrogen was higher with sunflower seed. Milk cholesterol content did not differ. Compared to sunflower seed diets, sunflower oil alone or in combination with fish oil showed positive effects on production parameters of late lactation dairy cows. 


\section{Milk Fatty Acid Profile}

The fatty acid composition of milk fat in the pre-trial and experimental treatments is shown in Table 8.

Table 8. Milk fatty acid (FA) profile of grazing dairy cows supplemented with sunflower seed (SS), sunflower oil (SO), sunflower seed plus fish oil (SS-FO) and sunflower oil plus fish oil (SO-FO).

\begin{tabular}{|c|c|c|c|c|c|c|c|c|c|}
\hline \multirow[b]{2}{*}{ Fatty acid (g/100g) } & \multicolumn{7}{|c|}{ Treatment $^{(1)}$} & \multicolumn{2}{|c|}{$\mathrm{P}<{ }^{(2)}$} \\
\hline & Pre-trial ${ }^{(3)}$ & SS & SO & SS-FO & SO-FO & SEM & A & B & $\mathrm{A} \times \mathrm{B}$ \\
\hline$C_{6: 0}$ & 1.11 & 0.30 & 0.38 & 0.21 & 0.35 & 0.04 & 0.06 & 0.30 & 0.52 \\
\hline $\mathrm{C}_{8: 0}$ & 0.91 & 0.28 & 0.26 & 0.13 & 0.21 & 0.03 & 0.03 & 0.37 & 0.15 \\
\hline $\mathrm{C}_{10: 0}$ & 2.03 & 0.70 & 0.68 & 0.31 & 0.54 & 0.08 & 0.21 & 0.01 & 0.18 \\
\hline $\mathrm{C}_{10: 1}$ & 0.28 & 0.63 & 0.07 & 0.03 & 0.04 & 0.01 & 0.33 & 0.05 & 0.80 \\
\hline $\mathrm{C}_{12: 0}$ & 2.49 & 1.03 & 1.10 & 0.56 & 0.90 & 0.12 & 0.13 & 0.03 & 0.31 \\
\hline $\mathrm{C}_{12: 1}$ & 0.16 & 0.06 & 0.06 & 0.03 & 0.04 & 0.00 & 0.13 & 0.00 & 0.14 \\
\hline $\mathrm{C}_{14: 0}$ & 9.13 & 4.66 & 5.20 & 2.71 & 4.80 & 0.42 & 0.02 & 0.03 & 0.11 \\
\hline $\mathrm{C}_{14: 1}+$ iso $_{15}$ & 1.10 & 0.71 & 0.73 & 0.55 & 0.62 & 0.02 & 0.10 & 0.00 & 0.34 \\
\hline $\mathrm{C}_{15: 0}$ & 2.01 & 1.20 & 1.57 & 0.90 & 1.39 & 0.10 & 0.01 & 0.06 & 0.58 \\
\hline $\mathrm{C}_{15: 1}$ & 0.42 & 0.24 & 0.21 & 0.23 & 0.26 & 0.01 & 0.74 & 0.26 & 0.10 \\
\hline $\mathrm{C}_{16: 0}$ & 27.14 & $18.7 \mathrm{a}$ & $18.09 \mathrm{a}$ & $17.41^{\mathrm{a}}$ & $21.20 \mathrm{~b}$ & 0.47 & 0.01 & 0.10 & 0.00 \\
\hline $\mathrm{C}_{16: 1}$ & 3.10 & 1.98 & 2.36 & 2.22 & 2.76 & 0.17 & 0.03 & 0.10 & 0.63 \\
\hline $\mathrm{C}_{17: 0}$ & 0.78 & 0.57 & 0.55 & 0.58 & 0.57 & 0.01 & 0.50 & 0.61 & 0.86 \\
\hline $\mathrm{C}_{17: 1}$ & 0.52 & $0.27 b$ & $0.35 \mathrm{a}$ & $0.39^{\mathrm{a}}$ & $0.37^{\mathrm{a}}$ & 0.01 & 0.14 & 0.00 & 0.02 \\
\hline $\mathrm{C}_{18: 0}$ & 9.53 & 15.6 & 12.53 & 7.12 & 5.73 & 0.97 & 0.05 & 0.00 & 0.37 \\
\hline trans $-\mathrm{C}_{18: 1}{ }^{(4)}$ & 2.79 & $13.47 \mathrm{~b}$ & $14.31 \mathrm{~b}$ & $33.54^{\mathrm{a}}$ & $27.78 \mathrm{c}$ & 1.24 & 0.09 & 0.00 & 0.00 \\
\hline cis- $\mathrm{C}_{18: 1}$ & 28.20 & 28.37 & 30.38 & 16.60 & 16.96 & 0.87 & 0.22 & $<.00$ & 0.38 \\
\hline $\mathrm{C}_{18: 2}$ & 1.79 & 2.77 & 1.60 & 2.50 & 1.43 & 0.11 & $<.00$ & 0.11 & 0.63 \\
\hline $\mathrm{C}_{18: 3}$ & 0.63 & 0.58 & 0.48 & 0.86 & 0.87 & 0.04 & 0.30 & 0.00 & 0.25 \\
\hline \multicolumn{10}{|l|}{ CLA } \\
\hline cis-9, trans-11 & 1.39 & 2.62 & 3.11 & 3.66 & 4.18 & 0.41 & 0.26 & 0.04 & 0.97 \\
\hline cis-12, trans-10 & - & 0.09 & 0.10 & 0.08 & 0.08 & 0.02 & 0.85 & 0.63 & 0.89 \\
\hline$c i s-9, c i s-11$ & 0.06 & 0.05 & 0.06 & 0.09 & 0.08 & 0.00 & 0.62 & 0.04 & 0.25 \\
\hline trans-9, trans-11 & 0.07 & 0.10 & 0.12 & 0.12 & 0.18 & 0.01 & 0.04 & 0.05 & 0.32 \\
\hline Total CLA & 1.52 & 2.82 & 3.38 & 3.96 & 4.51 & 0.42 & 0.23 & 0.03 & 0.99 \\
\hline $\mathrm{C}_{16: 0}+\mathrm{C}_{16: 1}$ & 30.24 & $20.6 \mathrm{a}$ & $20.45^{\mathrm{a}}$ & $19.63 \mathrm{a}$ & $24.00 \mathrm{~b}$ & 0.62 & 0.01 & 0.09 & 0.01 \\
\hline $\mathrm{C}_{20: 5 \mathrm{n}-3}(\mathrm{EPA})$ & 0.02 & 0.03 & 0.023 & 0.06 & 0.05 & 0.00 & 0.23 & 0.01 & 0.45 \\
\hline $\mathrm{C}_{22: 6 \mathrm{n}-3}(\mathrm{DHA})$ & - & 0.03 & 0.023 & 0.08 & 0.06 & 0.01 & 0.30 & 0.01 & 0.88 \\
\hline De novo FA $\left(\mathrm{C}_{4: 0}-\mathrm{C}_{15: 1}\right)$ & 19.63 & 9.21 & 10.30 & 5.60 & 9.19 & 0.74 & 0.02 & 0.01 & 0.14 \\
\hline Preformed FA $\left(>C_{17: 0}\right)$ & 45.92 & $64.56 \mathrm{a}$ & $63.46^{\mathrm{a}}$ & $65.60 \mathrm{a}$ & $58.36 \mathrm{~b}$ & 1.30 & 0.01 & 0.17 & 0.05 \\
\hline $\mathrm{CLA} /$ trans $-\mathrm{C}_{18: 1}$ & 0.5 & 0.21 & 0.21 & 0.10 & 0.15 & 0.01 & 0.22 & 0.00 & 0.20 \\
\hline $\mathrm{AI}^{(5)}$ & 1.88 & $0.80 \mathrm{~b}$ & $0.80 \mathrm{~b}$ & $0.50 \mathrm{a}$ & $0.80 \mathrm{~b}$ & 0.05 & 0.02 & 0.02 & 0.03 \\
\hline
\end{tabular}

${ }^{(1)}$ Least squares means \pm standard error of the mean (SEM). ${ }^{(2)}$ Duncan's Multiple Range Test. A $=(\mathrm{SS}+\mathrm{SS}-\mathrm{FO}) \mathrm{vs}(\mathrm{SO}+\mathrm{SO}-\mathrm{FO}) ; \mathrm{B}=(\mathrm{SS}+\mathrm{SO}) \mathrm{vs}(\mathrm{SS}-\mathrm{FO}+$ SO-FO). ${ }^{(3)}$ Pre-trial FA concentration before the start of sunflower seed. Sunflower oil or fish oil supply ( $\mathrm{n}=4$ cows). ${ }^{(4)}$ Trans-11 C18:1 represented $91.62 \%$, $89.66 \%, 91.93 \%, 94.46 \%$ of total trans- $\mathrm{C}_{18: 1}$ in SS, SO, SS-SO and SO-FO respectively. ${ }^{(5)} \mathrm{AI}$ : Atherogenicity Index $\left[\left(\mathrm{C}_{12: 0}+4 \mathrm{C}_{14: 0}+\mathrm{C}_{16: 0}\right) / \sum\right.$ unsaturated FA)]. 
The inclusion of both sources of linoleic acid (sunflower seed or oil) alone or combined with fish oil decreased the concentration of de novo synthesized fatty acids. The reduction in the more atherogenic saturated fatty acids averaged $63 \%$ for $\mathrm{C}_{12: 0}, 51 \%$ for $\mathrm{C}_{14: 0}$ and $29 \%$ for $\mathrm{C}_{16: 0}$. Compared to the pre-trial value of 1.88, the atherogenicity index of milk was reduced by supplementary PUFA, particularly when sunflower seed was combined with fish oil. Pre-trial concentration of $\mathrm{C}_{18: 0}$ was increased $(+51 \%)$ by both sources of $\mathrm{C}_{18: 2}$ (sunflower seed and oil) but it was decreased $(-31 \%)$ by fish oil. Pre-trial concentration of total trans $-\mathrm{C}_{18: 1}$ $(2.79 \%)$ was increased by supplemental lipids. Trans- $\mathrm{C}_{18: 1}$ was higher with fish oil (30.7\%) than the average value observed with sunflower seed and oil (13.9\%), and particularly when compared to sunflower seed-fish oil (significant $\mathrm{A} \times \mathrm{B}$ interaction). VA represented $91.62 \%, 89.66 \%, 91.93 \%$ and $94.46 \%$ of the total trans- $\mathrm{C}_{18: 1}$ in sunflower seed, sunflower oil, sunflower seed-sunflower oil and sunflower oil-fish oil respectively. The high trans- $\mathrm{C}_{18: 1}$ concentration induced by fish oil decreased the CLA/trans- $\mathrm{C}_{18: 1}$ ratio in the sunflower seed-fish oil and sunflower oil-fish oil treatments. The increase of 9-cis 11-trans CLA over basal (pre-trial) concentration $(1.39 \mathrm{~g} / 100 \mathrm{~g} \mathrm{FA})$ averaged $144 \%$ across treatments. No differences in milk CLA content were detected between both sources of supplementary $\mathrm{C}_{182^{2}}$. (sunflower seed or oil). Fish oil supply increased $(+37 \%)$ the average CLA content from 2.86 to $3.92 \mathrm{~g} / 100 \mathrm{~g} F A$ and increased the $\mathrm{C}_{18: 3 n-3}$. (sunflower seed or oil). Feeding sunflower seed increased milk $\mathrm{C}_{18: 2}$ content (2.64\%) compared to sunflower oil supply (1.52\%) without $\mathrm{AxB}$ interaction. fish oil supply increased concentrations of EPA (+97\%) and DHA $(+137 \%)$ in milk without effects of $\mathrm{C}_{18: 2}$ source. Secretion of individual fatty acids in milk fat is presented in Table 9.

Milk secretion of de novo synthesized fatty acids resulted lower in cows receiving sunflower seed (17.4 g/d) compared to those fed sunflower oil $(29.2 \mathrm{~g} / \mathrm{d})$ and also in cows supplemented with fish oil $(18.8 \mathrm{~g} / \mathrm{d})$ than in those without this supplement $(27.7 \mathrm{~g} / \mathrm{d})$. Milk secretion of $\mathrm{C}_{12: 0}$ resulted lower in sunflower seed $(1.73 \mathrm{~g} / \mathrm{d})$ compared to sunflower oil $(2.71 \mathrm{~g} / \mathrm{d})$ and also when cows were supplemented with fish oil ( 1.70 vs. $2.74 \mathrm{~g} / \mathrm{d})$. Milk secretion of $\mathrm{C}_{14: 0}$ resulted lower in sunflower seed $(7.69 \mathrm{~g} / \mathrm{d})$ instead of sunflower oil $(13.44 \mathrm{~g} / \mathrm{d})$ and also when fish oil was included in the diet ( 8.87 vs $11.95 \mathrm{~g} / \mathrm{d}$ ). The most effective combination in order to reduce $\mathrm{C}_{14: 0}$ secretion was sunflower seed-fish oil. Feeding sunflower seed reduced the $\mathrm{C}_{16: 0}$ secretion without effects of fish oil on this fatty acid. When sunflower seed was combined with fish oil the lowest milk secretion of $\mathrm{C}_{16: 0}$ was observed. In the present trial, the most useful treatment to reduce daily secretion of the atherogenic fatty acids $\left(\mathrm{C}_{12: 0}, \mathrm{C}_{14: 0}\right.$ and $\left.\mathrm{C}_{16: 0}\right)$ was sunflower seed combined with fish oil. Feeding fish oil significantly reduced the daily secretion of $\mathrm{C}_{18: 0}$ in milk. Yield of trans- $\mathrm{C}_{18: 1}$ acids resulted lower in sunflower seed (40.7 $\mathrm{g} / \mathrm{d})$ compared to sunflower oil $(57.1 \mathrm{~g} / \mathrm{d})$ treatment. Fish oil supply significantly increased secretion of trans- $\mathrm{C}_{18: 1}(64.5 \mathrm{vs.} 33.2 \mathrm{~g} / \mathrm{d})$. Total CLA secretion in milk was higher using free sunflower oil $(12.1 \mathrm{~g} / \mathrm{d})$ compared to sunflower seed (6.49 
Table 9. Fatty acid secretion in milk fat from grazing dairy cows supplemented with sunflower seed (SS), sunflower oil (SO), sunflower seed plus fish oil (SS-FO) and sunflower oil plus fish oil (SO-FO).

\begin{tabular}{|c|c|c|c|c|c|c|c|c|}
\hline \multirow[b]{2}{*}{ Fatty acid (g/d) } & \multicolumn{5}{|c|}{ Treatment $^{(1)}$} & \multicolumn{3}{|c|}{$\mathrm{P}<{ }^{(2)}$} \\
\hline & SS & SO & SS-FO & SO-FO & SEM & A & $\mathrm{B}$ & $\mathrm{A} \times \mathrm{B}$ \\
\hline $\mathrm{C}_{6: 0}$ & 0.71 & 1.20 & 0.18 & 1.02 & 2.25 & 0.04 & 0.22 & 0.52 \\
\hline $\mathrm{C}_{8: 0}$ & 7.72 & 0.75 & 0.22 & 0.60 & 0.11 & 0.14 & 0.03 & 0.21 \\
\hline $\mathrm{C}_{10: 0}$ & 1.77 & 1.92 & 0.53 & 1.54 & 0.30 & 0.10 & 0.03 & 0.19 \\
\hline $\mathrm{C}_{10: 1}$ & 0.17 & 0.20 & 0.04 & 0.12 & 0.03 & 0.22 & 0.03 & 0.45 \\
\hline $\mathrm{C}_{12: 0}$ & 2.53 & 2.95 & 0.93 & 2.47 & 0.37 & 0.04 & 0.03 & 0.19 \\
\hline $\mathrm{C}_{12: 1}$ & 0.14 & 0.17 & 0.05 & 0.13 & 0.02 & 0.05 & 0.02 & 0.31 \\
\hline $\mathrm{C}_{14: 0}$ & $10.82^{\mathrm{a}}$ & $13.80^{\mathrm{a}}$ & $4.57 \mathrm{~b}$ & $13.08^{\mathrm{a}}$ & 1.32 & 0.01 & 0.04 & 0.08 \\
\hline $\mathrm{C}_{14: 1}+$ iso $_{15}$ & 1.57 & 2.04 & 0.90 & 1.74 & 0.28 & 0.05 & 0.13 & 0.53 \\
\hline $\mathrm{C}_{15: 0}$ & 2.60 & 4.00 & 1.48 & 3.72 & 0.34 & 0.00 & 0.08 & 0.26 \\
\hline $\mathrm{C}_{15: 1}$ & 0.58 & 0.61 & 0.37 & 0.73 & 0.08 & 0.06 & 0.66 & 0.09 \\
\hline $\mathrm{C}_{16: 0}$ & $41.05 \mathrm{a}$ & $47.42 \mathrm{a}$ & $28.21 \mathrm{a}$ & $56.70 \mathrm{~b}$ & 5.25 & 0.01 & 0.74 & 0.08 \\
\hline $\mathrm{C}_{16: 1}$ & 4.56 & 5.97 & 3.56 & 7.35 & 0.65 & 0.01 & 0.78 & 0.11 \\
\hline $\mathrm{C}_{17: 0}$ & 1.24 & 1.52 & 0.93 & 1.55 & 0.21 & 0.08 & 0.54 & 0.46 \\
\hline $\mathrm{C}_{17: 1}$ & 0.64 & 0.89 & 0.62 & 1.00 & 0.08 & 0.01 & 0.61 & 0.47 \\
\hline $\mathrm{C}_{18: 0}$ & 34.36 & 33.81 & 11.50 & 15.01 & 6.64 & 0.83 & 0.02 & 0.76 \\
\hline trans $-\mathrm{C}_{18: 1}$ & 27.58 & 38.90 & 53.83 & 75.20 & 6.40 & 0.04 & 0.00 & 0.46 \\
\hline$c i s-\mathrm{C}_{18: 1}$ & 64.35 & 79.62 & 26.54 & 46.52 & 10.32 & 0.13 & 0.01 & 0.82 \\
\hline $\mathrm{C}_{18: 2}$ & 6.46 & 4.34 & 3.90 & 4.05 & 0.76 & 0.24 & 0.11 & 0.18 \\
\hline $\mathrm{C}_{18: 3}$ & $1.30^{\mathrm{a}}$ & $1.24^{\mathrm{a}}$ & $1.40 \mathrm{~b}$ & $2.42 \mathrm{~b}$ & 0.21 & 0.06 & 0.02 & 0.04 \\
\hline \multicolumn{9}{|l|}{ CLA } \\
\hline cis-9, trans- 11 & 6.05 & 9.64 & 6.05 & 12.9 & 1.81 & 0.02 & 0.40 & 0.40 \\
\hline cis-12, trans-10 & 0.14 & 0.29 & 0.13 & 0.23 & 0.08 & 0.17 & 0.70 & 0.74 \\
\hline cis-9, cis-11 & 0.05 & 0.11 & 0.14 & 0.21 & 0.03 & 0.05 & 0.01 & 0.9 \\
\hline trans- 9, trans- 11 & 0.21 & 0.40 & 0.21 & 0.50 & 0.08 & 0.03 & 0.56 & 0.50 \\
\hline Total CLA & 6.45 & 10.43 & 6.54 & 13.87 & 1.96 & 0.02 & 0.40 & 0.42 \\
\hline$C_{16: 0}+C_{16: 1}$ & $45.62^{\mathrm{a}}$ & $53.40^{\mathrm{a}}$ & $31.77 \mathrm{a}$ & $64.04 \mathrm{~b}$ & 5.83 & 0.01 & 0.80 & 0.08 \\
\hline $\mathrm{C}_{20: 5 \mathrm{n}-3}(\mathrm{EPA})$ & $0.08^{\mathrm{a}}$ & $0.06^{\mathrm{a}}$ & $0.09 \mathrm{~b}$ & $0.16 \mathrm{~b}$ & 0.02 & 0.16 & 0.023 & 0.06 \\
\hline $\mathrm{C}_{22: 6 \mathrm{n}-3}(\mathrm{DHA})$ & 0.08 & 0.06 & 0.13 & 0.20 & 0.03 & 0.48 & 0.02 & 0.21 \\
\hline De novo FA (4:0-15:1) & 24.48 & 31.03 & 10.38 & 27.28 & 3.37 & 0.01 & 0.03 & 0.17 \\
\hline Preformed FA (>17:0) & 142.50 & 170.90 & 105.50 & 160.01 & 21.40 & 0.10 & 0.30 & 0.56 \\
\hline
\end{tabular}

${ }^{(1)}$ Least squares means \pm standard error of the mean $(\mathrm{SEM}) .{ }^{(2)}$ Duncan's Multiple Range Test. A $=(\mathrm{SS}+\mathrm{SS}-\mathrm{FO})$ vs $(\mathrm{SO}+\mathrm{SO}-\mathrm{FO}) ; \mathrm{B}=(\mathrm{SS}+\mathrm{SO})$ vs $(\mathrm{SS}-\mathrm{FO}+$ SO-FO).

g/d) with significant increases due to supplementary fish oil. Total secretion of $n-$ 3 acids $\left(\mathrm{C}_{18: 3 n-3}\right.$, EPA and DHA) was significantly increased after fish oil supply.

\section{Rumen Environment and CP and NDF Degradation}

Interaction effects for parameters of in situ $\mathrm{CP}$ and NDF degradation were not 
detected (Table 10). The NDF fraction of the pasture showed a high rate and extent of digestion. Parameters of pasture NDF digestion did not differ between sunflower seed or oil even when fish oil was included. The source of linoleic acid (seed or sunflower oil) did not change the estimated soluble CP fraction of pasture. This parameter was increased from $31.3 \%$ to $49.6 \%$ when fish oil was added to the diets. The degradable fraction of $\mathrm{CP}$ was not affected by the source of linoleic acid, but tended to decrease by fish oil. Rate of pasture protein digestion was very high and similar across treatments. A significant treatment effect for the ruminal $\mathrm{pH}$ values was detected without interaction between treatment and hour of sampling. The highest mean was observed in sunflower seed-fish oil combination $(\mathrm{pH}=6.07)$ when compared to sunflower oil-fish oil $(\mathrm{pH}=5.77)$ without differences respect to sunflower seed $(\mathrm{pH}=5.84)$ and sunflower oil $(\mathrm{pH}=$ 5.81). Comparing free sunflower oil or sunflower seed with or without the addition of fish oil showed no changes in parameters of forage NDF and CP degradation in late lactation dairy cows grazing a fresh high quality forage.

\section{Discussion}

\subsection{Pasture and Diet Characteristics}

Pasture DM content (Table 3) was higher than values of $18 \%$ proposed as limitant for maximum DM intake for dairy cows [13]. Pasture CP content (Table 3) was in the range proposed by [14] to obtain high values of forage DM digestibility. NDF in pasture (Table 3 ) and in the whole rations (33\% to $36 \%$, Table 6) were near to the values (34\% - 36\%) suggested by [15] as no limitant for voluntary intake due to rumen fill. The mainly precursors of CLA are linoleic (present in corn silage, cereal grains, sunflower and soybean seeds) and $\alpha$-linolenic acids which is abundant in well managed leafy pastures and linseeds [16]. According

Table 10. Parameters of the in situ digestion of pasture neutral detergent fiber (NDF) and crude protein (CP) in grazing dairy cows supplemented with sunflower seed (SS), sunflower oil (SO), sunflower seed plus fish oil (SS-FO) and sunflower oil plus fish oil (SO$\mathrm{FO})$.

\begin{tabular}{cccccccccc}
\hline & \multicolumn{4}{c}{ Treatment $^{(1)}$} & \multicolumn{5}{c}{$\mathrm{P}<{ }^{(2)}$} \\
\cline { 2 - 9 } & SS & SO & SS-FO & SO-FO & SEM & A & B & A $\times$ B \\
\hline NDF & & & & & & & & \\
Degradable fraction, \% & 76.79 & 78.41 & 75.95 & 77.52 & 1.95 & 0.44 & 0.67 & 0.98 \\
Rate of digestion \%/h & 5.8 & 6.4 & 6.4 & 6.5 & 0.3 & 0.32 & 0.34 & 0.59 \\
CP & & & & & & & & \\
Soluble fraction, \% & 31.33 & 31.29 & 49.59 & 49.62 & 7.3 & 0.99 & 0.04 & 0.99 \\
Degradable fraction, \% & 62.15 & 51.42 & 42.95 & 44.74 & 6.73 & 0.53 & 0.10 & 0.38 \\
Rate of digestion, \%/h & 22 & 21 & 14 & 16 & 4.77 & 0.94 & 0.22 & 0.83 \\
\hline
\end{tabular}

${ }^{(1)}$ Least squares means \pm standar error of the mean $(\mathrm{SEM}) .{ }^{(2)}$ Duncan's Multiple Range Test $\mathrm{A}=(\mathrm{SS}+\mathrm{SS}-\mathrm{FO})$ vs $(\mathrm{SO}+\mathrm{SO}-\mathrm{FO}) ; \mathrm{B}=(\mathrm{SS}+\mathrm{SO})$ vs $(\mathrm{SS}-\mathrm{FO}+\mathrm{SO}-\mathrm{FO})$. 
to these observations, corn grain, corn silage and sunflower meal resulted rich in linoleic acid (49\%, 35\% and 64\% respectively) and in a less manner in oleic acid (32\%, 28\% and $20 \%$ respectively) whereas pasture represented a good source of $\alpha$-linolenic acid (56.5\%, Table 4). The EE content (7.64\%) resulted high if compared to the values recorded in a previous experiment (1.15 to $4.52 \% \mathrm{DM}$ ) [6] and also than those observed in the experiments by [17] [18] but in the normal range (3\% to $8 \%$ ) for high quality pastures [19]. In this trial, pasture content of linolenic acid (56\%) was also superior to the value (42\%) observed by [17] [18] but near to the value informed by [20]. The milk CLA enriching effects of pastures are mainly explained by a high linolenic acid intake, its conversion to trans-11 $\mathrm{C}_{18: 1}$ at ruminal level and the subsequent conversion by mammary cells to cis-9, trans-11 CLA from the enzymatic delta-9 desaturation of rumen derived VA [21]. It is worth noting that estimated pasture intake represented only 37 to $41 \%$ of total DM intake (Table 5) but the basal concentration of cis-9, trans-11 CLA in milk fat ( $1.43 \mathrm{~g} / 100 \mathrm{~g}$ FA) resulted high (Table 8 ). The daily strip-grazing system used coupled with a high leaf content of the oat sward and the relatively low efficiency of grazing (51\%) may have elicited the cow's selectivity for greenleaf materials contributing to explain the high basal cis-9, trans-11 CLA content of milk. Linoleic acid content in sunflower oil (55.2\%) was lower to mean values (68.5\%) reported by [4] but near to the $59.8 \%$ informed by [17]. Linoleic content of sunflower seed $(70.3 \%)$ resulted higher than observed in sunflower oil (Table 4). This fact induced a higher total estimated linoleic acid intake (+19\%) in treatments that included sunflower seed $(770 \mathrm{~g} / \mathrm{d})$ respect to sunflower oil $(646$ $\mathrm{g} / \mathrm{d}$ ) (Table 5). In the present experiment, sunflower oil represented about $5.6 \%$ of total DMI, linoleic acid $4.8 \%$ and linolenic acid about $0.87 \%$. The final oil concentration in the total ration may be considered as elevated because response in milk CLA content to sunflower oil feeding seems to be linear up to $4 \%$ oil in the total ration [1]. Concentration of fish oil in total DM intake averaged $1.6 \%$ (Table 5). The $\mathrm{C}_{16: 0}$ concentration in fish oil (19\%) was higher than values (12 to $16 \%)$ observed in other experiments [18] [22] [23]. The $\mathrm{C}_{18: 2 \mathrm{n}-6}$ concentration resulted particularly high (17\%) if compared to values of $0.8 \%$ to $1.6 \%$ informed by [18] [22]. Concentration of $\mathrm{C}_{18: 3 n-3}(1.49 \%)$ in fish oil compared well to values $(0.6 \%-3.76 \%)$ reported in [18] [22]. Concentration of EPA was extremely low $(6.71 \%)$ and out of the range of $13.9 \%-21.5 \%$ reported by [18] [22] [23] whereas DHA content (11\%) was within the range (14.2\% to $7.1 \%)$ observed in other trials [18] [22] [23].

\subsection{Milk Production and Composition}

As it was previously reported [5] [24], supplementation to dairy cows with PUFA did not affect milk yield (Table 7). Feeding $1.4 \mathrm{~kg}$ of sunflower seed to grazing dairy cows did not alter milk production $(25.2 \mathrm{~kg} / \mathrm{d})$ compared to control (25.3 kg/d) cows [25]. Replacing corn grain by sunflower seed (2 kg/d) did not increase milk or FCM compared to pre-trial records in dairy cows grazing a 
winter oat pasture [6]. Milk yield and milk fat content tended to increase when oilseeds rather than vegetable oils were fed to not grazing dairy cows [26] but such positive effects were not detected when lipid treatments were compared to control rations without oils seeds [25] [26] [27]. In our experiment, milk yield tended to be higher (13\%) in sunflower oil treatments (Table 7) a result that differed from [26]. The higher milk lactose content observed in sunflower oil treatments may have contributed to explain this result owing to its essential role as osmoregulator of milk volume. Feeding sunflower oil $(459 \mathrm{~g} / \mathrm{d})$ induced a non-significant increase in milk yield of about $1 \mathrm{~kg} / \mathrm{d}$ over control cows but that increase averaged only $0.7 \mathrm{~kg} / \mathrm{d}$ when the oil $(503 \mathrm{~g} / \mathrm{d})$ was fed "packed" in sunflower seed [28]. As milk fat content did not differ between sunflower seed and oil the higher FCM and milk fat secretion observed in sunflower oil treatments were mainly explained by the increase in milk yield (Table 7). Feeding fish oil had no effect on milk yield (Table 7) as observed in [28].

Milk fat content was very low in all groups (19.1 to $27.8 \mathrm{~g} / \mathrm{kg}$, Table 7) if compared to the average pre-trial content of $35.7 \mathrm{~g} / \mathrm{kg}$. Since negative effects of supplemental lipids on ruminal fiber digestion were not detected (Table 10) a direct effect on fat synthesis in the mammary gland by supplemental PUFA or trans-fatty acids formed during the ruminal biohydrogenation and ulterior transfer to the udder (Table 8) is the more likely explanation. The uptake of some specific preformed fatty acids (e.g. trans-10, cis-12 CLA and trans-8, cis-10 CLA) reduce the activity and/or expression of genes that encode important enzymes involved in uptake, synthesis and desaturation of fatty acids in the mammary gland [29]. Negative correlations between milk fat percentage and milk fat content of trans- $\mathrm{C}_{18: 1}(-0.65)$, CLA $(-0.63)$, EPA $(-0.67)$ and DHA (-0.58) were reported by [18]. Duodenal infusion of the trans-10, cis-12 CLA, an isomer that was increased in milk fat after lipid supplementation (Table 8) has been shown to reduce lipid secretion in milk $(-7 \%$ to $-36 \%)$ coupled with a short $\left(\mathrm{C}_{4: 0}\right.$ to $\left.\mathrm{C}_{8: 0}\right)$ and medium $\left(\mathrm{C}_{10: 0}\right.$ to $\left.\mathrm{C}_{16: 0}\right)$ chain fatty acids reduction [28]. Sunflower oil $(459 \mathrm{~g} / \mathrm{d})$ reduced milk fat concentration $(-3.4 \mathrm{~g} / \mathrm{kg}$ compared to control, $\mathrm{n}=5)$ whereas $503 \mathrm{~g} / \mathrm{d}$ of sunflower oil packed in sunflower seed showed no effect $(\mathrm{n}=$ 8 ) in the experiments reviewed by [28].

Marine oils negatively affect concentration and yield of milk fat [4]. Feeding fish oil $(305 \mathrm{~g} / \mathrm{d})$ to dairy cows reduced milk fat content $(-9.1 \mathrm{~g} / \mathrm{kg})$ and milk fat yield $(-208 \mathrm{~g} / \mathrm{d})$ in the 27 trials revised by [28]. Milk fat content was reduced from $34.8 \mathrm{~g} / \mathrm{kg}$ in control cows up to $29.9 \mathrm{~g} / \mathrm{kg}$ and $23.4 \mathrm{~g} / \mathrm{kg}$ in cows receiving 160 and $320 \mathrm{~g} / \mathrm{d}$ of fish oil respectively and milk fat yield decreased from 0.92 $\mathrm{kg} / \mathrm{d}$ to 0.78 and $0.58 \mathrm{~kg} / \mathrm{d}$ in supplemented cows [18]. In our experiment further reductions in milk fat content or yield in treatments that included fish oil were not detected (Table 7). Compared to pre-trial concentration, an important decrease in total de novo synthesized fatty acids was observed $(-106.2 \mathrm{~g} / \mathrm{kg}$, Table 9). This decrease was not apparently compensated with the increase in exogenous fatty acid uptake and milk fat content was reduced (Table 7). In non-grazing 
trials, protected oil supply showed a positive effect $(+0.64 \mathrm{~g} / 100 \mathrm{~g})$ on milk fat content [24] whereas in grazing cows PUFA supply appeared to have a negative effect on milk fat percentage [5]. Feeding oilseeds to non-grazing dairy cows showed inconsistent responses on milk fat content [26].

Results from grazing studies suggest that fat supplementation did not significantly reduce milk protein content with an increase in milk protein yield explained by concomitant increases in milk production [5] [30]. In our study, pre-trial milk protein concentration averaged $37.6 \mathrm{~g} / \mathrm{kg}$ and the protein/fat ratio ranged from 0.91 to 1.01. After unsaturated lipid supplementation, milk protein content remained high (35.4 to $40.7 \mathrm{~g} / \mathrm{kg}$ ) with a higher protein/fat ratio (1.57 to 1.94, Table 7) explained by the important decrease observed in milk fat in all treatments. Synthesis of milk protein can be limited by energy availability and the reduced milk fat content observed could improve energy status of the cows. The higher milk yield and protein concentration in sunflower oil cows explained the difference in milk protein yield $(75 \mathrm{~g} / \mathrm{d}, p<0.01)$ compared with sunflower seed treatments without significant effect of fish oil. In the experiments reviewed by [28] milk protein content decreased after feeding sunflower oil $(-1.1 \mathrm{~g} / \mathrm{kg}, \mathrm{n}=$ 5) and fish oil $(-1.2 \mathrm{~g} / \mathrm{kg}, \mathrm{n}=27)$ but not after sunflower seed supply $(\mathrm{n}=8)$. Feeding sunflower seed $(1.4 \mathrm{~kg} / \mathrm{d})$ to grazing cows slightly decreased $(-0.6 \mathrm{~g} / \mathrm{kg})$ milk protein percentage without effects on milk protein yield [25]. Milk protein content was lower in grazing cows receiving $3 \mathrm{~kg} / \mathrm{d}$ of rapeseed $(33.9 \mathrm{~g} / 100 \mathrm{~g})$ or soybean $(33.8 \mathrm{~g} / 100 \mathrm{~g})$ compared to control cows $(34.9 \mathrm{~g} / 100 \mathrm{~g})$ without effects on total milk protein secreted [27]. In grazing dairy cows, concentration or secretion of milk protein were not affected by a low dose $(160 \mathrm{~g} / \mathrm{d})$ of fish oil but both parameters were decreased by feeding $320 \mathrm{~g} / \mathrm{d}$ of fish oil [18].

\subsection{Milk Fatty Acid Composition}

Compared with pre-trial records (Table 8), PUFA supply decreased the hypercholesterolemic fraction of milk $\left(\mathrm{C}_{12: 0}, \mathrm{C}_{14: 0}\right.$ and $\left.\mathrm{C}_{16: 0}\right)$. Adding soybean or sunflower oil $(0.5 \mathrm{~kg} / \mathrm{d})$ to the concentrate of grazing dairy cows also resulted in decreases of short chain (35\%), medium chain fatty acids (20\%) and $\mathrm{C}_{12: 0}, \mathrm{C}_{14: 0}$ and $\mathrm{C}_{16: 0}(22 \%)$ compared to control [17]. Feeding fish oil to grazing dairy cows (160 or $320 \mathrm{~g} / \mathrm{d}$ ) reduced $\mathrm{C}_{12: 0}$ concentration in milk without any effect on $\mathrm{C}_{14: 0}$ and $\mathrm{C}_{16: 0}$ [18]. In our study, the sunflower seed-fish oil treatment was the most effective to reduce concentration of $\mathrm{C}_{16: 0}$ and $\mathrm{C}_{14: 0}$ and hence the $\mathrm{AI}$ of milk fat (Table 8). Significant reductions in $C_{6: 0}$ to $C_{10: 0}$ (Table 8) were also observed by [18] after fish oil feeding to grazing dairy cows. Comparing with pre-trial, feeding sunflower seed and sunflower oil enhanced concentration and secretion of $\mathrm{C}_{18: 0}$ (51\%) in milk fat (Table 8 and Table 9) reflecting increased ruminal biohydrogenation of supplementary $\mathrm{C}_{18: 2}$ and VA. The higher $\mathrm{C}_{18: 0}$ concentration in milk was observed in treatments that included sunflower seed respect to those including sunflower oil (Table 8). This result could be explained by the higher $\mathrm{C}_{18: 2}$ intake from sunflower seed (Table 5) and/or a greater rumen biohydrogenation 
due to a slower release of $\mathrm{C}_{18: 2}$ from sunflower seed as was previously observed in goats [28]. In the grazing experiment of [17] a lower dose $(0.5 \mathrm{~kg} / \mathrm{d})$ of oils (soybean or sunflower oil) did not modify milk basal concentration of $\mathrm{C}_{18: 0}(11.86$ $\mathrm{g} / 100 \mathrm{~g}$ vs 12.49 and $12.71 \mathrm{~g} / 100 \mathrm{~g}$ respectively). The lower concentration and secretion of $\mathrm{C}_{18: 0}$ in milk fat observed in both treatments that included fish oil (Table 8 and Table 9) may indicate an inhibition of biohydrogenation of supplementary $\mathrm{C}_{18: 2}$ (and also $\mathrm{C}_{18: 3}$ from pasture) in the rumen by EPA, DHA and their intermediates [23] [28]. A reduction in milk content of $C_{18: 0}$ from 11.97 to $7.68 \mathrm{~g} / 100 \mathrm{~g}$ was also observed after supplementation with $320 \mathrm{~g} / \mathrm{d}$ of fish oil to grazing cows [18]. In our trial, the absence of interaction between $\mathrm{C}_{18: 2}$ source and fish oil supply on milk $\mathrm{C}_{18: 0}$ concentration (Table 8) suggest that fish oil was equally effective in preventing exhaustive biohydrogenation to stearic acid with both sources of supplementary $\mathrm{C}_{18: 2}$.

Spreadability of milk fat could be improved by increasing the cis-9 $\mathrm{C}_{18: 1} / \mathrm{C}_{18: 0}$ ratio. This ratio resulted maximal during the pre-trial period (3.22, Table 8). The high contents of ether extract (7.64\% of DM) and $\mathrm{C}_{18: 3}$ (56.4\% of fatty acids) in the pasture (Table 4) and/or a higher activity of the mammary desaturase could explain that result. When the lipid content of the pasture was only $3 \%$ $4 \%$ with $42 \%$ to $50 \%$ of $\mathrm{C}_{18: 3}$ the cis- $9 \mathrm{C}_{18: 1} / \mathrm{C}_{18: 0}$ ratio in milk was comprised between 1.93 to 1.97 [17] [18] e.g. 40\% lower to than observed in the present experiment. After PUFA supplementation, the higher cis- $9 \mathrm{C}_{18: 1} / \mathrm{C}_{18: 0}$ ratio was observed in treatments that included fish oil (sunflower seed-fish oil $=2.33$ and sunflower oil-fish oil $=2.95$, Table 8 ) suggesting a partial rumen hydrogenation of supplementary $\mathrm{C}_{18: 2}$. It can also be speculated that inhibition by trans isomers of delta-9 mammary desaturase activity on $\mathrm{C}_{18: 0}$ was also low. Feeding $0.5 \mathrm{~kg}$ of sunflower or soy oils to grazing cows [17] increased oleic acid concentration (20\%) in milk with a cis-9 $\mathrm{C}_{18: 1} / \mathrm{C}_{18: 0}$ ratio of 2.17 that resulted lower to the values observed here (Table 8). fish oil alone reduced stearic and oleic acid concentration in milk fat without changes in the basal $\mathrm{C}_{18: 1} / \mathrm{C}_{18: 0}$ ratio (1.97) at low $\left(160 \mathrm{~g} / \mathrm{d} ; \mathrm{C}_{18: 1} / \mathrm{C}_{18: 0}=1.94\right)$ or high $\left(320 \mathrm{~g} / \mathrm{d}, \mathrm{C}_{18: 1} / \mathrm{C}_{18: 0}=2.04\right)$ doses of FO [18].

In our grazing experiment a high basal concentration of total CLA (1.57 g/100g FA) and 9-cis, 11-trans CLA (1.43 g/100g FA) was observed (Table 8) although herbage allowance was moderate $(11 \mathrm{kgDM} / \mathrm{cow})$ and pasture intake averaged only $39 \%$ of total DM intake of cows (Table 6). After 17 weeks of grazing, milk CLA content was dependent on herbage allowance with the lower values observed at $16 \mathrm{~kg}$ pasture $\mathrm{DM} /$ cow/d compared with $20 \mathrm{~kg} \mathrm{DM} /$ cow/d [30]. In our experiment, the high basal milk CLA concentration may be the consequence of elevated contents of lipid and $\mathrm{C}_{18: 3}$ in pasture (Table 4) suggesting that these parameters may be determinant factors even at moderate levels of pasture intake. With a lower pasture lipid (3\% - 4\%) and $\mathrm{C}_{18: 3}(42 \%-50 \%)$ contents, basal CLA resulted $12 \%$ to $20 \%$ lower than values observed here averaging $1.26 \mathrm{~g} \mathrm{CLA} / 100 \mathrm{~g}$ FA although pasture intake was not informed by the authors [17] [18]. At a similar pasture lipid content, the highest concentration of milk 
CLA $(2.21 \mathrm{~g} / 100 \mathrm{~g}$ FA) was observed when pasture represented $100 \%$ of total DM intake compared to $33 \%(0.89 \mathrm{~g} / 100 \mathrm{~g} \mathrm{AG})$ or $66 \%(1.43 \mathrm{~g} / 100 \mathrm{~g} \mathrm{AG})$ [31].

When the main objective is to increase milk CLA content, feeding vegetable oils rich in PUFA $\left(\mathrm{C}_{18: 2}\right.$ or $\left.\mathrm{C}_{18: 3}\right)$ is very effective. At equally doses added to the ration, fish oil is more effective than plant oils at increasing the CLA concentration in milk [1]. Milk CLA content was $2.02 \mathrm{~g} / 100 \mathrm{~g}$ by feeding soy or sunflower $(0.5 \mathrm{~kg} / \mathrm{d})$ oils [17] but it increased to 3.23 and $3.64 \mathrm{~g} / 100 \mathrm{~g}$ when 0.16 or 0.32 $\mathrm{kg} / \mathrm{d}$ of fish oil were added to the ration of grazing cows [18]. The results obtained here (Table 8) confirmed those observations indicating that the combination of supplementary linoleic acid (sunflower seed or oil) with fish oil is a natural way for farmers to increase the 9-cis, 11-trans and total CLA in milk fat even when basal CLA concentration is high. Feeding fish oil alone (160 and $320 \mathrm{~g} / \mathrm{d}$ ) to grazing dairy cows yielded concentrations of trans $-\mathrm{C}_{18: 1}$ in milk of 8.53 and $11.96 \mathrm{~g} / 100 \mathrm{~g}$ FA [18] values that resulted $61 \%$ to $72 \%$ lower than observed in our trial when sunflower seed or sunflower oil were combined with fish oil (Table 8).

In grazing dairy cows, direct comparisons to test the advantage of feeding lipids in the form of seeds or free oils in order to limit rumen hydrogenation and maximize milk CLA content are scarce. In the present experiment, the ruminal dose-pulse model via ruminal cannulae was used to simulate a rapid intake of $\mathrm{C}_{18: 2}$ by the cow. It revealed no differences in milk fat concentration of trans- $\mathrm{C}_{18: 1}$ or CLA when sunflower seed was compared to sunflower oil (Table 8). The average milk content of cis-9, trans-11 CLA in PUFA supplemented cows (3.39 $\mathrm{g} / 100 \mathrm{~g}$ FA) resulted $137 \%$ higher than pre-trial basal values (Table 8 ). This CLA concentration resulted also higher than values reviewed by [4] but near to values (3.65 to $3.7 \mathrm{~g} \mathrm{CLA} / 100 \mathrm{~g} \mathrm{FA}$ ) informed by [32] in non-grazing diets. In our trial, basal milk CLA content (Table 8) resulted 226\% higher than values observed in the grazing trials reviewed by [22] and $128 \%$ higher than milk CLA content after PUFA supplementation [29]. Milk CLA content in supplemented cows was also $68 \%$ higher to those obtained after feeding $0.5 \mathrm{~kg} / \mathrm{d}$ of sunflower or soybean oils (2.02 g CLA/100g FA) in grazing cows by [18]. In spite of the moderate herbage allowance and pasture intake of cows responses to supplementary PUFA in milk CLA concentrations resulted 53\% higher than the maximal CLA levels reported by [31] in $100 \%$ pasture diets. It is possible that not only the lipid content of pasture but other pasture components might alter rumen biohydrogenation producing a synergistic effect with PUFA supplements.

Total concentration of trans- $\mathrm{C}_{18: 1}$ in milk resulted high particularly in treatments that included fish oil (Table 8). This increase over pre-trial values was expected as a strong correlation exists between the concentration of CLA and trans- $\mathrm{C}_{18: 1}$ not only in milk but also in beef muscle. This is an important concern because intake of trans fatty acids is associated with incidence of cardiovascular disease in humans. However, it was postulated that ruminant trans fatty acids, especially concerning the effect on cardiovascular risk, do not possess the same 
unfavorable effects as industrially produced trans fatty acids [33]. The presence of VA in dairy products may also have positive health properties through its direct anticarcinogenic properties [34] or mediated through its endogenous conversion to cis-9, trans-11 CLA by tissue delta-9 desaturases in humans [35]. The metabolisation of VA to CLA has been shown to prevent chemically induced carcinogenesis in rodents [36]. In our experiment, milk trans-11 $\mathrm{C}_{18: 1}$ represented from $89.7 \%$ to $94.5 \%$ of total trans-C18:1 in the four treatments.

The observed reduction in CLA/trans $-\mathrm{C}_{18: 1}$ ratio in fish oil treatments suggests that further reduction of trans- $\mathrm{C}_{18: 1}$ intermediates was effectively inhibited. The lack of differences in milk fat concentration between treatments with or without fish oil (Table 7) and the higher milk CLA content observed (Table 8) also suggest that any putative shift from trans-11 $\mathrm{C}_{18: 1}$ to trans-10 $\mathrm{C}_{18: 1}$ induced by fish oil was probably not occuring. Measured in four cows per treatment from the whole experiment, the ratio trans-11 $\mathrm{C}_{18: 1} /$ trans-10 $\mathrm{C}_{18: 1}$ increased when fish oil was included from 9.2 (sunflower seed) and 8.6 (sunflower oil) to 11.5 (sunflower seedfish oil) and 17.2 (sunflower oil-fish oil). It is possible that a very high trans-11 $\mathrm{C}_{18: 1}$ flux to the mammary gland may have exceeded the desaturation capacity in mammary cells or that specific fatty acids contained in fish oil may have inhibited the delta-9 desaturase activity as reported by [28].

In the present experiment, fish oil represented about $1.6 \%$ of total DM intake of cows (Table 6). Maximal concentration of trans-11-C $\mathrm{C}_{18: 1}$ and CLA in milk was observed when fish oil represented about $2 \%$ of total DM intake of cows [37] [38] without further increments when fish oil was increased up to $3 \%$ of total DM intake [38]. It was also stated that fish oil can be reduced at $1 \%$ of total DM intake when it is combined with other sources of lipid substrate [39]. The optimal doses of precursors $\left(\mathrm{C}_{18: 2}\right.$ or $\left.\mathrm{C}_{18: 3}\right)$ for CLA synthesis and its combination with fish oil in order to maximize the milk CLA content and the CLA/trans$11 \mathrm{C}_{18: 1}$ ratio needs to be explored in grazing dairy cows.

In spite of the high supplementary $\mathrm{C}_{18: 2}$ doses used in this experiment, its incorporation to milk fat appeared to be moderated (sunflower seed treatments) or null (sunflower oil treatments) if compared to pre-trial $\mathrm{C}_{18: 2}$ concentrations (Table 8). This result was consistent with that observed by [17] and suggests a moderate efficiency in the transfer of dietary $\mathrm{C}_{18: 2}$ to milk fat and a high rate of rumen biohydrogenation. In our experimental conditions, sunflower seed instead of sunflower oil behave as a more effective carrier of $\mathrm{C}_{18: 2}$ in order to increase its concentration in milk fat ( $2.64 \%$ vs $1.52 \%$ of $\mathrm{C}_{18: 2}$ respectively).

Milk concentration of EPA and DHA were increased by fish oil but the absolute increment should be considered low (EPA $=0.03$ and DHA $=0.04 \mathrm{~g} / 100 \mathrm{~g}$ FA). The result may be explained in part by the low EPA and DHA contents in fish oil as well as by the high rumen biohydrogenation of these fatty acids as observed by [1] [28]. It was also postulated that EPA and DHA are present in the cholesteryl esthers and plasmatic phospholipids fractions that are poorly utilized by the mammary gland [1] [40]. The low transfer effectiveness of EPA and DHA 
from the diet to the milk is consistent with previous findings [1] [18].

\subsection{Rumen Environment and In Situ Kinetics of CP and NDF Degradation}

In our trial, dietary sources of PUFA showed to be very effective to modulate milk fatty acids composition decreasing the atherogenic fatty acids and increasing milk CLA content and hence adding healthy value to milk. For the farmer, it is also important to avoid ruminal metabolic disorders in supplemented cows in order to sustain milk yield and cows health. The use of oils rich in PUFA may decrease ruminal cellulolysis and reduce performance of cows if some negative effects on pasture NDF and CP digestion are induced. This could in turn decrease DM intake of cows and energy content of rations putatively decreasing milk production. In our experiment, the rate and extent of the NDF fraction of the pasture did not differ between sources of supplementary $\mathrm{C}_{18: 2}$ used (sunflower seed or sunflower oil) nor by fish oil (Table 10). Absolute values for parameters of NDF degradation were although lower than those observed in nonsupplemented cows from another grazing study using a similar pasture [41]. In this later experiment, adding $2 \mathrm{~kg}$ of sunflower seed increased the rate of pasture NDF degradation from $7.5 \% / \mathrm{h}$ to $9.1 \% / \mathrm{h}(\mathrm{p}<0.02)$ without any effect on the degradable NDF fraction between control (89.02\%) and sunflower seed (81.98\%) supplemented cows [41]. Experimental data concerning the effects of fat supplementation on rumen digestion in grazing trials are scarce. In the five experiments revised by [5] no negative effects of supplemental saturated and unsaturated fat on ruminal NDF digestion were observed. The in situ grazing experiments performed at INTA Balcarce showed that the rate of digestion and the effective degradability of pasture NDF were not affected by saturated or unsaturated fat supplements [5]. Those results agree with the observation that negative effects are minimal in diets with a high proportion of forage [42]. The high rate of passage from dairy cows grazing high quality pastures [29] and the high levels of calcium of these pastures [5] could alleviate any negative effect of fat supplements. The soluble CP fraction of pasture was increased when fish oil was added to diets whereas the degradable fraction of CP tended to be lower (Table 10) without changes in rate of $\mathrm{CP}$ digestion. A higher undegradable protein balance may have contributed to alleviate the detrimental effects on milk protein content often observed when fish oil is fed to dairy cows. The targeted nutritional approaches used here (sunflower oil or sunflower seed with or without the addition of fish oil) showed no detrimental effects in parameters of forage NDF and CP degradation in late lactation dairy cows grazing a fresh high quality forage.

\section{Conclusion}

Milk produced from cows grazing high quality pastures may be a good source of natural CLA even when the herbage allowance was low $(11 \mathrm{~kg} \mathrm{DM} / \mathrm{d})$ and pasture intake represented only $37 \%$ to $41 \%$ of total DM intake. This result could be 
explained by the high lipid and $\mathrm{C}_{18: 3}$ contents in the high quality pasture utilized. In these conditions, supplementary PUFA deeply affected milk fatty acid composition reducing the potential hypercholesterolemic fatty acids, the AI of milk fat and milk fat content. These healthy properties of milk fat were mainly achieved by feeding sunflower seed combined to fish oil. Independently of the supplemental source of $\mathrm{C}_{18: 2}$ (sunflower seed or sunflower oil), feeding fish oil reduced content of $\mathrm{C}_{18: 0}$ in milk and increased that of trans- $\mathrm{C}_{18: 1}$ and CLA indicating a reduction in biohydrogenation of trans $-\mathrm{C}_{18: 1}$ to $\mathrm{C}_{18: 0}$ in the rumen. At about $1.61 \%$ of total DM intake, fish oil combined to sunflower oil or sunflower seed apparently exceeded the desaturation capacity of the mammary gland to convert trans-C18:1 to CLA leading to a high trans-C18:1 concentration. Although the natural trans isomers of $\mathrm{C}_{18: 1}$ are probably not themselves harmless and VA can be metabolized by humans to CLA, its intake should be maintained low by humans until the effects on human health became well known. Considering that natural trans-isomers of CLA are health-promoting and play specific beneficial roles in humans, the optimal combination between fish oil and PUFA $\left(\mathrm{C}_{18: 2}\right.$ and $\left.\mathrm{C}_{18: 3}\right)$ sources should be determined in grazing dairy cows before recommending dietary modifications in order to maximize milk CLA and CLA/ trans-11 $\mathrm{C}_{18: 1}$ ratio. The changes in the milk fatty acid profile induced by treatments confirmed the high plasticity of milk fat composition and should contribute to alleviate the dieticians criticism againts dairy products and to improve the health quality of milk and its image as perceived by the consumer. The results obtained here should to be confirmed using natural means of supplementing PUFA by mixing oil sources to feedstuffs in grazing dairy cows.

\section{References}

[1] Chilliard, Y., Ferlay, A., Mansbridge, R.M. and Doreau, M. (2000) Ruminant Milk Fat Plasticity: Nutritional Control of Saturated, Polyunsaturated, Trans and Conjugated Fatty Acids. Annales de Zootechnie, 49, 181-205. https://doi.org/10.1051/animres:2000117

[2] Ulbritch, T.L. and Southgate, D.A.T. (1991) Coronary Heart Disease: Seven Dietary Factors. Lancet, 338, 985-992. https://doi.org/10.1016/0140-6736(91)91846-M

[3] Parodi, P.W. (1999) Conjugated Linoleic Acid and Other Anticarcinogenic Agents of Bovine Milk Fat. Journal of Dairy Science, 82, 1339-1349. https://doi.org/10.3168/jds.S0022-0302(99)75358-0

[4] Stanton, C., Murphy, J., McGrath, E. and Devery, R. (2003) Animal Feeding Strategies for Conjugates Linoleic Acid Enrichment of Milk. In: Sébédio, J.L., Christie, W.W. and Adloff, R., Eds., Advances in Conjugated Linoleic Acid in Food, AOCS Press, Champaign, 123-145.

[5] Schroeder, G.F., Gagliostro, G.A., Bargo, F., Delahoy, J.E. and Muller, L.D. (2004) Effects of Fat Suplementation on Milk Production and Composition by Dairy Cows on Pasture: A Review. Livestock Production Science, 86, 1-18. https://doi.org/10.1016/S0301-6226(03)00118-0

[6] Gagliostro, G.A., Schang, M.E., Garciarena, D.A., Fernández, H.H., Guaita, M.S. and Páez, R. (2004) Reemplazo de Grano de Maíz por Semilla de Girasol en Vacas 
Lecheras en Pastoreo: 1. Consumo de Materia Seca, Producción y Composición de la Leche. Revista Argentina de Producción Animal, 24, NA 32 (In CD).

[7] Meijs, J.A.C., Walters, R.J.K. and Keen, A. (1982) Sward Methods. In: British Grassland Society, Ed., Herbage Intake Handbook, British Grassland Society, Nantwich, 11-36.

[8] Goering, H.K. and Van Soest, P.J. (1970) Forage Fiber Analysis (Apparatus Reagents, Procedures and Some Applications). Agriculture Handbook. United States Department of Agriculture, Washington DC.

[9] Dische, Z. (1962) General Color Reactions. In: Whistler, R.L. and Wolfram, M.L., Eds., 187 Carbohydrate Chemistry, Academic Press, New York, 477-512.

[10] Association of Official Agricultural Chemists (1995) Official Methods of Analysis. 16th Edition, Association of Official Agricultural Chemists, Washington DC, Chapter $4,17$.

[11] Macrae, J.E. and Armstrong, D.G. (1968) Enzyme Method for Determination of Alpha-Linked Glucose Polymers in Biological Materials. Journal of the Science of Food and Agriculture, 19, 578-581. https://doi.org/10.1002/jsfa.2740191006

[12] Ørskov, E.R. and McDonald, I. (1979) The Estimation of Protein Degradability in the Rumen from Incubation Measurements Weighted According to Rate of Passage. Journal of Agricultural Science, 92, 499-503. https://doi.org/10.1017/S0021859600063048

[13] Verité, R. and Journet, M. (1970) Influence de la Teneur en eau et de la Déshydratacion de l'Herbe sur Sa Valeur Alimentaire pour les Vaches Laitirès. Annales de Zootechnie, 10, 269-277.

[14] Minson, D.J. (1990) Forage in Ruminant Nutrition. Academic Press, Inc., Samford Valley.

[15] Mertens, D.R. (1994) Regulation of Forage Intake. In: Jur, F., Ed., Forage Quality. Evaluation and Utilization, American Society of Agronomy, Madison, 59-114.

[16] Chilliard, Y., Ferlay, A., Loor, J., Rouel, J. and Martin, B. (2002) Trans and Conjugated Fatty Acids in Milk from Cows and Goats Consuming Pasture or Receiving Vegetable Oils or Seeds. Italian Journal of Animal Science, 1, 243-254. https://doi.org/10.4081/ijas.2002.243

[17] Rego, O.A., Rosa, H.J.D., Portugal, P.V., Franco, T., Vouzela, C.M., Borba, E.S. and Bessa, R.J.B. (2005) The Effects of Supplementation with Sunflower and Soybeans Oils on the Fatty Acid Profile of Milk Fat from Grazing Dairy Cows. Animal Research, 54, 17-24. https://doi.org/10.1051/animres:2005002

[18] Rego, O.A., Rosa, H.J.D., Portugal, P., Cordeiro, R., Borba, A.E.S., Vouzela, C.M. and Bessa, R.J.B. (2005) Influence of Dietary Fish Oil on Conjugated Linoleic Acid, Omega-3 and Other Fatty Acid in Milk Fat from Grazing Dairy Cows. Livestock Production Science, 95, 27-33. https://doi.org/10.1016/j.livprodsci.2004.11.040

[19] Harfoot, C.G. (1981) Lipid Metabolim in the Rumen. In: Christie, W.W., Ed., Lipid Metabolism in Ruminant Animals, Pergamon Press, New York, 21-56. https://doi.org/10.1016/B978-0-08-023789-3.50006-4

[20] Agenäs, S., Holtenius, K., Griinari, M. and Bursted, E. (2002) Effects of Turnout to Pasture and Dietary Fat Supplementation on Milk Fat Composition and Conjugated Linoleic Acid in Dairy Cows. Acta Agriculturae Scandinavica, Section A-Animal Sciences, 52, 25-33. https://doi.org/10.1080/09064700252806399

[21] Griinari, J.M. and Bauman, D.E. (1999) Biosynthesis of Conjugated Linoleic Acid and Its Incorporation into Meat and Milk in Ruminants. In: Yurawecz, M.P., Mos- 
soba, M.M., Framer, J.K.G., Pariza, M.W. and Nelson, G.J., Eds., Advances in Conjugated Linoleic Acid Research, AOCS Press, Champaign, 180-200.

[22] Loor, J.J., Doreau, M., Chardigny, J.M., Ollier, A., Sebedio, J.L. and Chilliard, Y. (2005) Effects of Ruminal or Duodenal Supply of Fish Oil on Milk Fat Secretion and Profiles of Trans-Fatty Acids and Conjugated Linoleic Acid Isomers in Dairy Cows Fed Maize Silage. Animal Feed Science and Technology, 119, 227-246. https://doi.org/10.1016/j.anifeedsci.2004.12.016

[23] Shingfield, K.J., Ahvenjarvis, T.V., Arola, A., Nurmela, K.U.V., Huhtanen, P. and Griinari, J.M. (2003) Effect of Dietary Fish Oil on Biohidrogenation of Fatty Acids and Milk Fatty Acids Content in Cows. Journal of Animal Science, 77, 165-179.

[24] Gagliostro, G.A. and Chilliard, Y. (1992) Revisión Bibliográfica. Utilización de Lípidos Protegidos en Nutrición de Vacas Lecheras. I. Efecto Sobre la Producción y la Composición de la Leche y Sobre la Ingestión de Materia Seca y Energía. Revista Argentina de Producción Animal, 12, 1-15.

[25] Rearte, D.H., Santini, F.J., García, P.T., Maritano, M. and Elizalde, J.C. (1989) Efectos de la Suplementación de Semilla de Girasol Sobre la Producción y Composición de la Leche. Revista Argentina de Producción Animal, 9, 6-12.

[26] Morand-Fehr, P., Chilliard, Y. and Bas, P. (1986) Répercusions de l'Apport de Matieres Grasses dans la Ration sur la Production et la Composition du Lait de Ruminant. Institut National de la Recherche Agronomique, 64, 59-72.

[27] Lawlees, F., Murphy, J.J., Harrington, D., Devery, R. and Stanton, C. (1998) Elevation of Conjugated Cis-9, Trans-11 Octadecadienoic Acid in Bovine Milk because of Dietary Supplementation. Journal of Dairy Science, 81, 3259-3267. https://doi.org/10.3168/jds.S0022-0302(98)75890-4

[28] Chilliard, Y. and Ferlay, A. (2004) Dietary Lipids and Forages Interactions on Cows and Goat Milk Acid Composition and Sensory Properties. Reproduction Nutrition Development, 44, 467-492. https://doi.org/10.1051/rnd:2004052

[29] Bargo, F., Muller, L.D., Kolver, E.S. and Delahoy, J.E. (2003) Invited Review: Production and Digestion of Supplemented Dairy Cows on Pasture. Journal of Dairy Science, 86, 1-42. https://doi.org/10.3168/jds.S0022-0302(03)73581-4

[30] Stanton, C., Lawless, F., Kjellmer, G., Harrington, D., Devery, R., Connolly, J.F. and Murphy, J. (1997) Dietary Influences on Bovine Milk Cis-9, Trans-11 Conjugated Linoleic Acid Content. Journal of Food Science, 62, 1083-1086.

https://doi.org/10.1111/j.1365-2621.1997.tb15043.x

[31] Dhiman, T.R., Anand, G.R., Satter, L.D. and Tariza, M.W. (1999) Conjugated Linoleic Acid Content of Milk from Cows Fed Different Diets. Journal of Dairy Science, 82, 2146-2156. https://doi.org/10.3168/jds.S0022-0302(99)75458-5

[32] Solomon, R., Chase, L.E., Ben Ghedalia, D. and Bauman, D.E. (2000) The Effect of Nonstructural Carbohydrate and Addition of Full Fat Extruded Soybeans on the Concentration of Conjugated Linoleic Acid in the Milk Fat of Dairy Cows. Journal of Dairy Science, 83, 1322-1329. https://doi.org/10.3168/jds.S0022-0302(00)74998-8

[33] Department of Health (1994) Nutritional Aspects of Cardiovascular Disease, Report of the Cardiovascular Review Group of the Committee of Medical Aspects of Food Policy (COMA). Report No. 46. His Majesty's Stationery Office, London.

[34] Awad, A.B., Hermann, T., Fink, C.S. and Horvath, P.J. (1995) 18:1 N7 Fatty Acids Inhibit Growth and Decreased Inositol Phosphate Release in HT-29 Cells Compared to N-9 Fatty Acids. Cancer Letters, 91, 55-61. https://doi.org/10.1016/0304-3835(95)03725-C 
[35] Parodi, P.W. (2003) Conjugated Linoleic Acid in Food. In: Sébédio, J.L., Christie, W.W. and Adloff, R., Eds., Advances in Conjugated Linoleic Acid in Food, AOCS Press, Champaign, 101-122. https://doi.org/10.1201/9781439822166.ch8

[36] Banni, S., Angioni, E., Murru, E., Carta, G., Melis, M.P., Bauman, D., Dong, Y. and Ip, C. (2001) Vaccenic Acid Feeding Increases Tissue Levels of Conjugated Linoleic Acid and Suppreses Development of Premalignant Lesions in Rat Mammary Gland. Nutrition and Cancer, 41, 91-97. https://doi.org/10.1080/01635581.2001.9680617

[37] Baer, R.J., Ryali, J., Schingoethe, D.J., Kasperson, K.M., Donovan, D.C., Hippen, A.R. and Franklin, S.T. (2001) Composition and Properties of Milk and Butter from Cows Fed Fish Oil. Journal of Dairy Science, 84, 345-353. https://doi.org/10.3168/jds.S0022-0302(01)74483-9

[38] Donovan, D.C., Schingoethe, D.J., Baer, R.J., Ryali, J., Hippen, A.R. and Franklin, S.T. (2000) Influence of Dietary Fish Oil on Conjugated Linoleic Acid and Other Fatty Acids in Milk Fat from Lactating Dairy Cows. Journal of Dairy Science, 83, 2620-2628. https://doi.org/10.3168/jds.S0022-0302(00)75155-1

[39] Ramaswamy, N., Baer, R.J., Schingoethe, D.J., Hippen, A.R., Kasperson, K.M. and Whitlock, L.A. (2001) Composition and Flavor of Milk and Butter from Cows Fed Fish Oil, Extruded Soybeans, or Their Combination. Journal of Dairy Science, 84, 2144-2151. https://doi.org/10.3168/jds.S0022-0302(01)74659-0

[40] Offer, N.W., Marsden, M., Dixon, J., Speake, B.K. and Thacker, F.E. (1999) Effect of Dietary Fat Supplements on Levels of N-3 Polyunsaturated Fatty Acids, Trans Acids and Conjugated Linoleic Acid in Bovine Milk. Journal of Animal Science, 69, 613625. https://doi.org/10.1017/S135772980005147X

[41] Garciarena, D., Fernández, H.H., Schang, M.E. and Gagliostro, G.A. (2004) Reemplazo de Grano de Maíz por Semilla de Girasol en Vacas Lecheras en Pastoreo. 3. Ambiente Ruminal y Digestión de la Pastura. Revista Argentina de Producción Animal, 24, NA 34 (In CD).

[42] Palmquist, D.L. (1984) Use of Fats in Diets for Lactating Dairy Cows. In: Wiseman, J., Ed., Fats in Animal Nutrition, Butterworths, London, 357-381. https://doi.org/10.1016/B978-0-408-10864-5.50025-5

Submit or recommend next manuscript to SCIRP and we will provide best service for you:

Accepting pre-submission inquiries through Email, Facebook, LinkedIn, Twitter, etc. A wide selection of journals (inclusive of 9 subjects, more than 200 journals)

Providing 24-hour high-quality service

User-friendly online submission system

Fair and swift peer-review system

Efficient typesetting and proofreading procedure

Display of the result of downloads and visits, as well as the number of cited articles

Maximum dissemination of your research work

Submit your manuscript at: http://papersubmission.scirp.org/

Orcontact as@scirp.org 